\title{
A Serious Gaming Approach for Crowdsensing in Urban Water Infrastructure with Blockchain Support
}

\author{
Alexandru Predescu*(D), Diana Arsene (D), Bogdan Pahonțu (D), Mariana Mocanu (D) and Costin Chiru (D) \\ Computer Science and Engineering Department, Faculty of Automatic Control and Computers, \\ Politehnica University of Bucharest, Splaiul Independenței 313, 060042 Bucharest, Romania; \\ arsene.diana.andreea@gmail.com (D.A.); pahontubogdan@gmail.com (B.P.); mariana.mocanu@upb.ro (M.M.); \\ costin.chiru@upb.ro (C.C.) \\ * Correspondence: alexandru.predescu@upb.ro
}

Citation: Predescu, A.; Arsene, D.; Pahonțu, B.; Mocanu, M.; Chiru, C. A Serious Gaming Approach for Crowdsensing in Urban Water Infrastructure with Blockchain Support. Appl. Sci. 2021, 11, 1449. https://doi.org/10.3390/app 11041449

Academic Editor: Marc Kurz Received: 30 December 2020 Accepted: 1 February 2021 Published: 5 February 2021

Publisher's Note: MDPI stays neutral with regard to jurisdictional claims in published maps and institutional affiliations.

Copyright: (c) 2021 by the authors. Licensee MDPI, Basel, Switzerland. This article is an open access article distributed under the terms and conditions of the Creative Commons Attribution (CC BY) license (https:/ creativecommons.org/licenses/by/ $4.0 /)$

\begin{abstract}
This paper presents the current state of the gaming industry, which provides an important background for an effective serious game implementation in mobile crowdsensing. An overview of existing solutions, scientific studies and market research highlights the current trends and the potential applications for citizen-centric platforms in the context of Cyber-Physical-Social systems. The proposed solution focuses on serious games applied in urban water management from the perspective of mobile crowdsensing, with a reward-driven mechanism defined for the crowdsensing tasks. The serious game is designed to provide entertainment value by means of gamified interaction with the environment, while the crowdsensing component involves a set of roles for finding, solving and validating water-related issues. The mathematical model of distance-constrained multi-depot vehicle routing problem with heterogeneous fleet capacity is evaluated in the context of the proposed scenario, with random initial conditions given by the location of players, while the Vickrey-ClarkeGroves auction model provides an alternative to the centralized task allocation strategy, subject to the same evaluation method. A blockchain component based on the Hyperledger Fabric architecture provides the level of trust required for achieving overall platform utility for different stakeholders in mobile crowdsensing.
\end{abstract}

Keywords: mobile crowdsensing; serious gaming; blockchain; urban water; gamification

\section{Introduction}

Cyber-Physical-Social systems (CPSS) are essential for sustainable developments such as smart grids, autonomous vehicles and health monitoring [1]. Mobile CrowdSensing (MCS) has untapped potential in water resources management for both data collection and raising awareness about water-related problems [2]. Citizen science is essential for this matter, to allow harnessing the potential of mobile devices in the scope of the community [3].

During the last few decades, modern society has evolved around the emerging technologies that have shaped the way we interact with the environment. The video game industry has created one of the leading technologies in digital entertainment. Video games have been developed since the beginning of the digital age, based on the major hardware and software advancements over the last few decades. From arcade games to home consoles and PC (Personal Computer) games, there is now a variety of platforms, genres and communities built around this cultural medium with global implications [4].

In this technology-driven world, Serious Gaming (SG) can harness the power of video games in transforming the way we interact with the environment and raising awareness about water-related problems. Such an example is represented by collaborative applications for increasing the level of participation of citizens in the community [5].

There are many challenges associated with achieving a sustainable development of a modern society that is both resource-efficient and ethical, of which the level of trust between the stakeholders is of primary importance. Blockchain technology was created as 
a solution to the problem of mistrust and has the ability to reconfigure industries by ensuring transparency and trust between network members, reducing excessive bureaucracy, reducing costs, facilitating faster transactions and improving the flow of these transfers [6]. In this sense, efficient management of water resources can be achieved by using blockchain as a trusted storage ledger shared between stakeholders [7].

Since considerable advancements have been made over the last few years in these emerging technologies, the scope of each piece of the puzzle must be carefully considered for the sustainable development of modern society [8,9].

The main objectives of this paper are: (1) to realize a scientific study of approaches related to serious games, focusing on applications for water resource planning and management; (2) to design a serious game in order to analyze and evaluate various scenarios in the context of water distribution systems; (3) to design and evaluate a secure storage layer based on blockchain technology.

\subsection{The Mobile Crowdsensing Paradigm}

In MCS, users share data from integrated sensors in their mobile devices, complementing the wide adoption of IoT (Internet of Things) devices. With the support of IoT infrastructure, opportunistic sensing can be integrated to take advantage of the ubiquity of personal devices, GPS and Mobile Networks [10].

Participatory sensing is a form of MCS, requiring a strategy for motivating the users, tapping into the social aspects (social networks) and gamification (game theory). Challenges include privacy and security (GPS, disclosure of location information), quality of data (low-quality sensors, number of participants, data evaluation), trustworthiness (trust by reliability for anonymous participants, trust by decision for trusted participants, reputation systems such as collaborative scores, vote-based), energy (battery level, influenced by device model, sensors, GPS and networking usage, sampling frequency), incentives (rewards for quality of data, increasing the involvement of participants), agent-based strategies (AR/VR) [11].

MCS frameworks assume an opportunistic assignment of sensing tasks, with reputationaware schemes that can have a significant effect on platform utility in the context of urban water reporting. Optimal user assignment according to the reputation and sensing cost is a challenge in MCS that involves geolocation, reliability (quality of sensing data, robust and secure services, encryption, truthful participation) and incentives (gamification, game theory, auction-based models, reward-based mechanisms) [12].

MCS requires an incentive scheme to be effective, while the amount and accuracy of sensing data should be based on the active participation of citizens. Some of the main challenges in robust MCS systems are given by the trust, reliability and security aspects (e.g., privacy protection, malicious behavior, security threats) of crowd-sensed data from anonymous sources [13]. Solutions include reputation systems (trust, reliability) and incentives (increasing participation, compensating for privacy concerns). In this sense, an optimization problem can be formulated based on the level of trust and reward-driven mechanism, while the data exchange and storage requirements should ensure an optimal security scheme that ensures the required level of trust for service providers.

Game theory can be applied to define the incentive model where multiple players are involved and the interaction between players in the form of cooperative and noncooperative games. Incentive mechanisms can be defined for crowdsensing in the context of a CPSS, such as the classification of users based on the level of trust [14], while different incentive schemes for MCS can be classified in terms of platform-centric and user-centric approaches [15].

\subsection{The Evolution of Modern Gaming}

The history of video games began with the precursor to the arcade game "Pong", made on a CRT (cathode ray tube) display with analog controls. During the 1980s and 1990s, the golden age of video games was defined by timeless classics such as Pac-Man, Space 
Invaders, Mario Bros and Tetris, and many of the classic game genres were defined such as FPS (First Person Shooter), RTS (Real-Time Strategy) and RPG (Role-Playing Game).

Nonetheless, the core mechanics of modern games can be attributed to the rich cultural heritage of our society. Since antiquity, games have been an important cultural medium, capturing the essence of different civilizations and time frames, such as the Sumerian game called The Royal Game of Ur (4000 BC), the Chinese game Wei Qi (2200 BC), the Egyptian game Senet (1500 BC), the Scandinavian game Hnefatafl (400 AD), the Indian game Chaturanga (700 AD) and Persian game of Shatranj, the precursor of chess (600 AD) [16]. A brief history of video games can be defined by three major eras $[17,18]$ :

- The pre-crash era: During the 1970s, Atari dominated the arcade game industry with titles such as Pong (1972), Space Invaders (1978), Pac Man (1982) and Donkey Kong (1982), generating more revenue than both the pop music industry and film industry. Soon, the industry was growing too quickly to maintain the momentum. The downfall happened in 1983, when a game adaptation of the box office success, E.T., was rushed to market with a nearly impossible deadline. Due to the subsequent low quality and critical reception, it is considered one of the biggest commercial failures in video game history, which led to the collapse of Atari and the crash of the entire video game industry in 1983;

- The technology advancement race: With the release of Commodore 64 in 1982, the PC was becoming the new platform for gaming, as were home consoles (NES, Game Boy, Sega Mega Drive, PlayStation, Xbox), which led to a series of technological advancements that restarted the industry;

- The online boom: The rise of the internet and mobile technologies transformed the industry into what was to become the multi-billion-dollar industry with the highest growth among the entertainment industries, with an estimated 2.7 billion gamers worldwide.

Nowadays, video games are interactive cultural environments, encouraging active participation and creating a new sense of collaboration in a post-industrial society, where digital entertainment and virtual interaction are becoming more and more important $[19,20]$. Video games can provide a snapshot of past and present society, allowing the player to experience certain events, which can be directed towards educating the user about the world, such as managing a city and its infrastructure (e.g., SimCity), building empires (e.g., Age of Empires) and real-time strategy (e.g., World of Warcraft).

Over the past few decades, the video game industry has been characterized by an increase in both the number of new games and the level of realism that competes with that of the movie industry [21]. Video game magazines, websites, blogs and YouTube channels are an integral part of the video game culture, and user reviews and ratings have accelerated the development of the gaming industry, providing an interactive feedback loop. While cutting-edge graphics and realism are a defining aspect of modern video games, the user perspective is greatly influenced by past expectations, as the industry has reached a level of maturity.

As a paradox of modern gaming, video games quickly become obsolete with regards to technical advancements, while at the same time, the recent success of retro gaming is an indicator of user preference towards less sophisticated games [22].

Mobile gaming is an example of a different approach, focusing on simple yet innovative design rather than super-realistic gameplay. Before the smartphone age, the most popular early mobile phone game was Snake (1997) which was commonly found preinstalled on most Nokia phones. Early mobile internet (WAP) allowed for downloading mobile games, but this was a rather rudimentary, slow process, while more recent phones with USB connectivity allowed installing new games downloaded from the internet. It was not until the launch of the iPhone and App Store in 2007 and 2008 as well as the Android operating system and Google Play in 2008 that mobile games began to disrupt the traditional gaming industry. The mobile gaming industry now accounts for more than $45 \%$ 
of the global gaming market, with a high level of integration within the mobile ecosystem and business model innovation (e.g., in-app purchases, mobile ads) [23].

\subsection{The Role of Serious Gaming}

Mobile games have been at the forefront of some real-world experiments, such as location-based games, with the first attempt as early as 2001 (BotFighters, with cell location services and SMS messages). Treasure hunting and "geocaching", urban adventure games and massively multiplayer mobile games are some of the examples of mobile game disruption both in-game and in the real world.

The role of mobile devices as an extension with sociocultural dimensions can be seen in the modern world, especially in the Asia Pacific region, where the level of integration with mobile technologies is unprecedented [24].

On the other hand, serious games are becoming more and more pervasive, with applications in e-Health, e-Commerce and e-Learning, where gamification has become a key ingredient in increasing the level of interaction and motivation of users, providing incentives and game-like rewards [25].

SG analysis and design include the role of players in real-world scenarios, with a broad range of possibilities and specific limitations. In [26], a conceptual model based on Activity Theory provides a model of human behavior in their social and organizational context and the roles of different game components in achieving the high-level objectives from the perspective of educational purpose. A taxonomy of SG components is presented, where the game mechanics are extended to include the learning and instructional process involved in such games.

The SG industry is driven by analytics to support decision-making through actionable insights, e.g., learning analytics and game analytics. While gamification is an essential aspect in SG, the utility function is of primary importance. Such examples include simulation games for military training (e.g., Marine DOOM) or message broadcasting (e.g., America's Army) [27].

Research shows that serious games in the water sector are an essential key to discovering socio-technical-economic problems among the parties involved. The modeling, simulation and optimization tools used in water management systems are based on decision science [28] and were born during World War II, with the help of mathematicians and engineers, in order to support military operational planning $[29,30]$. These games have a similar history, appearing during the war. The earliest use of games used to support decision making are war games [30]. These are based on the planning of military operations.

In [31], Clark introduced the notion of serious games, being seen as simulation games used in various areas with major impacts on the population, such as education and decision making in public policymaking. Since then, many research studies have been done on this topic, in various fields of application, including water management [32-34]. Due to the multitude of third parties and areas involved in SG, different approaches and even conflicts arise. In [35], Gleick presents the fact that water was involved in various conflicts, including as a military target, as an object of terrorism or as a political tool. Solving these conflicts consists in computer modeling [29]. In this sense, the concept of shared vision planning [36] involves all third parties in the development and simulation of models reaching a consensus.

\subsection{Blockchain}

To reach a consensus, the required trust level between participants must be ensured. The problems with centralized frameworks arise when considering unauthorized attempts to collect information and active misbehavior of participants and stakeholders, with all these factors contributing to a decreasing level of trust and participation, thus having a negative impact on platform utility. Blockchain overcomes these limitations, greatly reducing the risk of illegitimate transactions and unauthorized disclosure of private information. Therefore, the problem of trust in a water resource management system can be solved 
considering the increased transparency enabled by blockchain technology and integrated consensus algorithms [7].

Approaches to water management vulnerabilities place more emphasis on regional or national areas, not taking into account the fact that water sources and demands are linked in the water network on a geographical scale [37]. Therefore, the approach of a distributed network such as blockchain technology can be beneficial for dealing with vulnerabilities on multiple levels $[38,39]$.

There are many definitions of blockchain technology. From a technical point of view, blockchain is a peer-to-peer distributed register that is cryptographically secure with the possibility of only adding information and immutable and that can be updated only by consensus of all nodes [40].

According to [41], blockchain is defined as a chain of blocks, and each block contains transactions that are recorded in a register that is distributed to all nodes in the network, as shown in Figure 1. Transactions remain in a chain and are immutable, and because the register is distributed to all participating nodes, any node can verify the transaction and announce the verification in order to reach a consensus. Much more simply, Andreas Antonopoulos defines blockchain as "The Internet of Money" in a publication with the same name [42].

Blockchain has become, through its security, a decentralized architecture and flexibility, one of the most important technologies of today. Researchers in almost all fields have tried to adapt this new technology and develop new solutions.

In the water industry, blockchain technology can have various implications such as: keeping records (with the help of smart contracts, companies can access data in real-time), reporting and audit (since the data and actions are registered in an immutable register, it offers the possibility of enhanced audit support), data reconciliation (allowing regulators to control and monitor the status of transactions) and digitizing water (the demand for freshwater requires effective resource management solutions) [43].

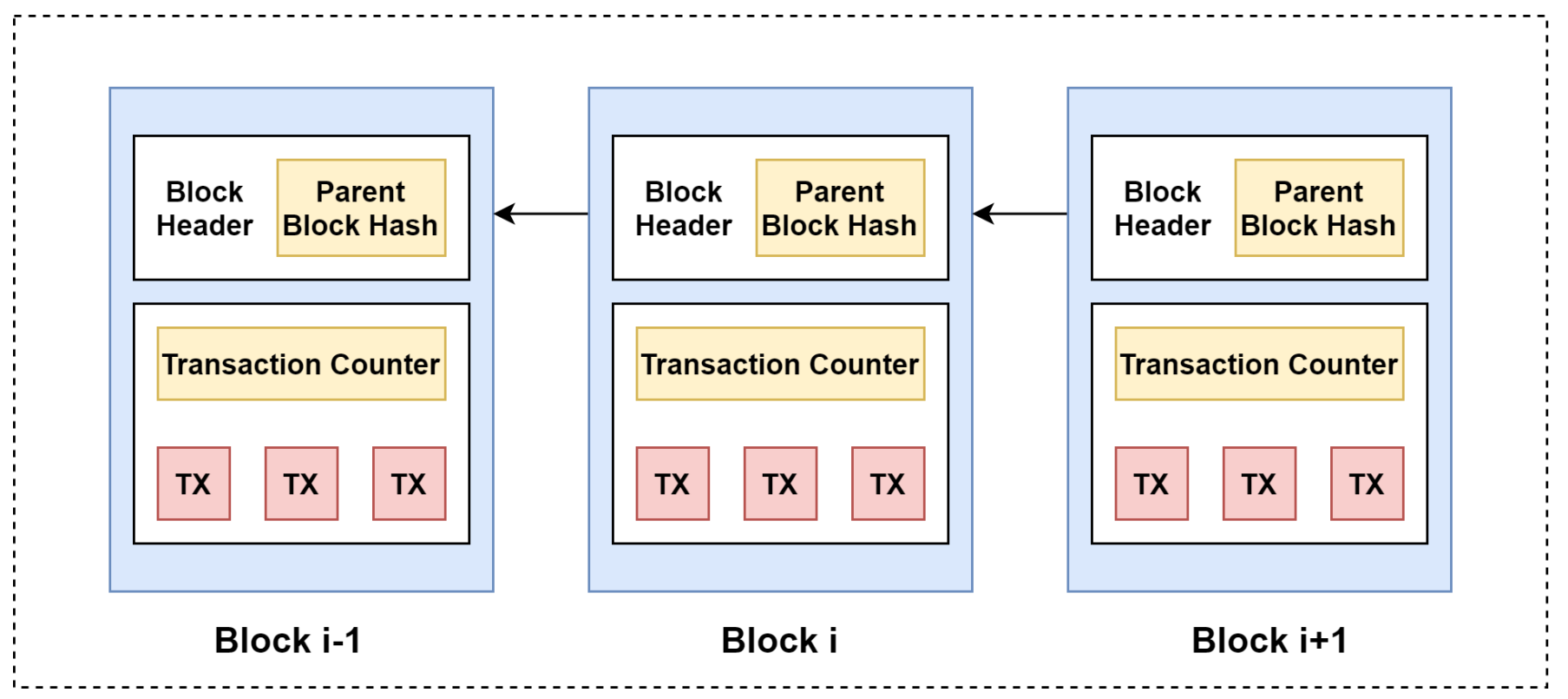

Figure 1. Blockchain architecture.

Current blockchain systems are classified into three types: public blockchains, private blockchains and consortium blockchains. In public blockchains, all records are visible to the public, and everyone could take part in the consensus process. In contrast, only a group of pre-selected nodes can participate in the consensus process of a consortium blockchain. Regarding the private blockchain, only the nodes coming from a specific 
organization are able to join the consensus process. The consortium blockchain built by several organizations is partially decentralized, because only a small part of the nodes are selected to determine the consensus. Consortium blockchains are commonly used in business applications [44].

In this sense, rules are implemented by smart contracts, which allow anonymous parties to interact, without the need for an intermediary [45]. The origin and history of smart contracts are much older than Bitcoin and date back to the 1990s. The term "smart contract" was first used in 1993 by one of the alleged creators of Bitcoin, Nick Szabo, and is referred to as a computer program that can automatically execute the terms of any contract or agreement [46].

The general steps by which a smart contract works in a distributed public register are represented by coding (introducing the appropriate logic in their writing, which cannot be altered), the public register (the contract is sent similarly to the creation of a new blockchain transaction), execution (a peer receives the code and executes it, then checks the execution of the code from other computers, reaching an agreement on the results).

The network will update the distributed registers to record the execution of the contract. In this type of system, manipulation by one party is not possible, because the control over the execution of the smart contract is performed in the distributed network, being validated by many participants [47].

There are multiple benefits of smart contracts, such as reducing trading time, increasing transparency, recording all terms and conditions in explicit detail, security based on the highest level of data encryption currently available, permanent storage and backup solution, eliminating the need for a wide range of intermediaries, and real-time verification [48].

While being a new technology, and with its implementation not entirely documented in practice, blockchain technology can prove to be useful and versatile for our world, as it can facilitate most systems in different industries.

\subsection{Paper Structure}

The paper is structured as follows. Section 2 presents the related work in MCS, SG and Blockchain, with implications for water resource management. Section 3 presents a data-driven survey of video games and mobile games, as well as related scientific papers to provide a real-world background for the proposed solution. In Section 4, we present the theoretical background, which precedes the proposed solution in Section 5, describing the three major components: a crowdsensing model, a blockchain model and the SG platform. In Section 6, the materials and methods for creating the experimental framework are described, while the results are presented in Section 7 for the crowdsensing and the blockchain models, in terms of accuracy and performance. For MCS, we aim to provide a location-independent evaluation of the proposed platform-centric and user-centric approaches, while the blockchain solution is tested for performance and scalability. Section 8 presents some general discussions based on the proposed solution and experimental results, compared with related studies, while Section 9 outlines the conclusions and future directions of work.

\section{Related Work}

In this section, related work in the domain of MCS, SG and Blockchain is presented in terms of the multiple approaches and solutions, with regards to water resource management and beyond.

\subsection{Mobile Crowdsensing}

Crowdsensing networks are an important part of the CPSS paradigm. In [2], a systematic literature review reveals an overview of search terms in Web of Science related to crowdsensing.

Existing studies in MCS focus on either the global approach or specific problems, based on theoretical models (game theory, information theory), applied sciences (data science, 
computational intelligence) and engineering (system architectures, mobile technologies, communication systems).

In [49], a review of technical approaches and applications in smartphone-based crowdsensing solutions precedes the proposed reference architecture that can be adapted to the various MCS architecture designs with the major components defined for client-side, server-side and the communications level.

Besides the technical complexity of MCS architectures, sustainable citizen involvement in MCS is difficult to achieve when considering sensing costs (battery, mobile data, time, effort) and long-term commitment with indirect benefits for participants.

In this sense, the Vehicle Routing Problem (VRP) is a highly studied optimization problem that can be applied for the allocation of tasks in an MCS solution that involves the optimization of sensing costs for smart urban mobility [50]. Transportation and traffic management are some of the domains where vehicle routing is a primary objective, while MCS proves to be essential for real-world applications [51].

While cost-efficient allocation of tasks is important for the overall system, an incentive mechanism should be considered for increasing the participation of citizens in solving largescale problems at the community level [5]. For this reason, there are many research papers addressing the problem of optimal incentives to achieve higher levels of participation [52].

Game theory was proposed in 1928 by John von Neumann, while incentive schemes include reputation-based schemes that promote cooperation, such as PETrust (penaltyincentive mechanism); Peer-to-Peer (P2P) networks, where trust plays an important role; and credit-based schemes, which promote cooperation by rewarding participants for helping others [53].

Morris and Shadmehr [54] define an optimization model for exogenous rewards and punishments in the context of a "regime change" game, considering the overall equilibrium, optimal effort and optimal reward schemes. The reward scheme can be determined based on the level of optimism for optimal participation of citizens. While offering higher rewards would induce a higher level of participation from otherwise less involved citizens, the level of participation of otherwise highly involved citizens would decrease. The optimal reward scheme requires adapting to the level of optimism of each citizen to achieve equilibrium.

Auction-based incentives are widely used in the context of MCS, requiring an allocation rule and a payment rule for sensing tasks, such as the Vickrey-Clarke-Groves (VCG) auction model. For achieving the overall utility of the platform, trust and reputation can be used as a reward scheme, based on the number of true and false reports for each participant.

Considering a user-centric approach, task assignment in MCS relies on the reported sensing costs, while VCG-based auction is used to achieve truthful reporting. The proposed solutions are commonly evaluated in terms of sensing coverage, incentive cost and truthfulness using given scenarios [55]. In this paper, we aim to provide a location-independent evaluation of VCG for truthful participant selection under different initial conditions in terms of geolocation.

VCG and proposed alternatives were used by Tao and Song [56] in MCS for efficient path planning and increasing the level of truthful participation. While the experimental setup is evaluated for a single distribution of tasks, in the current paper, we propose a more comprehensive simulation to evaluate the overall performance in real-world scenarios.

The VCG mechanism is actually quite flexible and can be applied in other domains, such as electricity markets $[57,58]$, public transportation/car-sharing systems $[59,60]$ or auction-based network routing [61,62].

Endorsements are another form of social incentive that involve sharing rewards within groups. Other incentive mechanisms that are less common in MCS include bargaining games, contract theory (principal-agent) and market-driven mechanisms (supply-demand interaction) [52].

Liu et al. [63] propose a hybrid incentive mechanism based on reputation and rewards, with an encryption algorithm scheme for privacy protection. While there are different 
types of incentives for MCS, such as monetary incentives, virtual currencies, gamification and social interactions, more recent approaches propose a hybrid incentive strategy [64].

$\mathrm{Xu}$ et al. [53] define an incentive mechanism in accordance with the level of cooperation of the participants, using an evolutionary game model based on the principles of game theory in economics and demonstrated in simulation. The strategy based on game theory allows for the optimization of a cost function that achieves a balance between rewarding participants and the data collection process.

An important aspect in urban crowdsensing is given by duplicate reporting, either intentional or accidental, with negative implications on platform utility, which requires either an accurate detection scheme or a reward adjustment scheme. While duplicate detection based on physical location alone may be inaccurate, Zhang and Wang [65] propose a detection scheme based on categorical, temporal and spatial information using an unsupervised learning algorithm.

\subsection{Serious Gaming for Water Resource Management}

This section presents the research done in the field of serious games, with applicability in water resource management. There are different game theory models, such as cooperative and non-cooperative (alliances/competitions), information games (decisions based on perfect/complete/incomplete information), evolutionary games (strategy updates, highly competitive), static and dynamic games (simultaneous/sequential decisions), zero-sum games (sum of the payoffs equals zero, e.g., poker, chess) [14].

A classification of games can be defined by different characteristics such as number of players, simultaneous/sequential, perfect information (player knows all previous moves), complete information (players know the structure of the game), zero-sum games (sum of the payoffs equals zero, e.g., poker, chess), communication, and cooperative/non-cooperative. Real-world games are rarely zero-sum games [66]. The limitations, requirements and conceptual frameworks for SG are discussed in [67].

Talasila et al. [68] propose a model for providing uniform sensing coverage by means of in-game incentives and geolocation, i.e., earning points for shooting alien invaders. Smartphone users are therefore encouraged to cover the entire target area, collecting data along the way. The results of the study show that mobile gaming ensures high coverage and increasing levels of player engagement. The effect of gamification on platform utility is twofold: providing uniform coverage and increasing player motivation to participate in crowdsensing activities (from $46 \%$ coverage with micro-payments to $87 \%$ coverage with gamification). Moreover, the placement and movement strategy for alien invaders was shown to have a significant effect on the platform efficiency, i.e., area coverage over time.

The literature contains an extensive analysis on this subject with regards to water resource management, trying to improve the consumption of water resources and to bring more awareness to the consumer while game design elements are used for increased participation $[5,69,70]$. The applications presented offer examples of good practice, as well as limitations and improvements that can be made in future development.

One of these games is SimCity ${ }^{\mathrm{TM}}$, which deals with decision-making regarding city building, including urban planning. This game is characterized by the simulation and integration of complex systems using agent-based modeling [71]. Among other functionalities, the water supply of the built city, its treatment and distribution are also taken into account.

Another serious game project is Aqua Republica [72], in which players have the opportunity to create a river basin and to make decisions on how to operate it. The consequences of the decisions can be rewarded or sanctioned, according to the models implemented in the game. This game was developed by DHI (Danish Hydraulic Institute) [73] and involves the achievement of three main objectives: (1) educating the player by being aware of how they make decisions; (2) developing a sustainable water management system through the exchange of knowledge; (3) providing prompt and optimal action in critical situations in order to not destabilize the proper functioning of water resources. A specific feature for 
this game is the combination of the game mode with the simulation mode, called MIKE Hydro Basin [74], offering players the opportunity to act as a manager of that river basin.

Chew et al. [75] propose a pilot solution in the Middle Oliphant region of South Africa. This game starts in the year 200, with certain input parameters such as population, funds, food and energy but also an unhealthy ecosystem. During each round, each player makes decisions about how the pool works, trying to keep the high parameters related to the sustainability of the pool.

Another serious game approach is called Shariva (Shared River) [76]. The main purpose of creating this game was to cooperate, communicate and raise awareness among stakeholders regarding transboundary river basins.

Another example of a SG is presented in [77] and consists in the development of ways to adapt to climate change on the rivers in the Netherlands. The aim of the game is to manage a virtual river, consisting of several players who are part of two different teams. The parties involved are either Dutch water managers or academics. Consensus also plays a key role in decision-making involving the stakeholders and several areas of activity.

Seibert and Vis [78] present a serious multiplayer game approach targeting farmers and water issues that may arise, which allows cooperation between players to observe differences between the collaborative and the individual behavior.

Bassi et al. [79] present the game WATERSTORY, which is based on an interactive learning environment (ILE) offering the parties involved the opportunity to raise awareness and exchange knowledge in the process of managing water demand on the island of Maui. The authors consider that this approach can achieve a consensus on how to manage water resources, with a minimum of conflicts and a maximum of objectives achieved, taking into account the rules imposed by the authorities.

The SimDelta Program is an extension of the Dutch Delta Program [80], being an interactive online simulation model based on policy development for freshwater supply and to avoid flooding in the Rhine-Meuse delta. However, Rijcken and Christopher [81] conclude that SimDelta is not really a serious game, although it fulfills some of the criteria, because users cannot interact with each other, but only with the experts who present the solution.

Another interesting approach to serious games is presented by Hill et al. [82], where the authors used the game to evaluate the decisions made in case of a drought. Participants were chosen from different fields, such as students, graduates and stakeholders in the water domain.

The presented research emphasizes the fact that serious games have been and are involved in the water sector, being an important tool in the understanding by third parties of socio-technical-economic problems in terms of water resource management. Lately, the integration of games with other complex systems has become mandatory for good awareness, cooperation and optimal results, while gamification in MCS for urban water reporting, such as defined in this paper, is not commonly found in related studies.

\subsection{Blockchain for Water Resource Management}

According to $\mathrm{Wu}$, Song and Wang [83], several algorithms for consensus can be used depending on the blockchain type: Proof of Work, Proof of Stake, Delegate Proof of Stake, Proof of Existence, Proof of Activity, Proof of Elapsed Time and Byzantine Fault Tolerance. One of the first used consensus algorithms is Proof of Work. Originally used in Bitcoin, Proof of Work continues to ensure very high security but with a high energy cost.

Sankar, Sindhu, and Sethumadhavan [84] performed a survey about consensus algorithms used in blockchain applications and presented the following technologies: Hyperledger Fabric, Corda and Stellar Consensus Protocol.

With the help of intelligent sensors, a large number of data can be collected. For integrating these modern technologies [85] to provide an increased level of trust, smart contracts can be used, which are based on secure and immutable data. This helps the validation process in an efficient way and supports decision making [86]. 
Bordel et al. [87] propose such a water control system, based on blockchain networks, where community members have the opportunity to establish rules regarding the use of water quotas. These rules are implemented through a smart contract [45]. The main challenge is to transform water control techniques into a distributed system that all members of the community can trust.

Given that in recent years, natural phenomena such as floods, droughts, tsunamis and hurricanes have increased significantly, government organizations have considered the development of smart, sustainable and safe infrastructure to monitor water consumption $[88,89]$. A particularly important problem is found in rural as well as urban areas and consists in ensuring the necessary quality and quantity of water [90]. This approach has been successfully implemented in Australia and Singapore [91-93]. In Australia, the South East Queensland (SEQ) water network was established for the ultimate purpose of managing and securing urban water supply [94].

The water supply network established in Singapore is based on water collection, management, recycling and distribution [94,95]. Intelligent sensors were used for real-time monitoring of water quality, as well as analytical tools to support decision-making systems, while the developed system ensured a sustainable and efficient management of the water supply network using blockchain technology [90].

In 2017, start-up Civic Ledger [96] realized a feasibility study related to the transparency of Australian water markets, with the aim of increasing the trust, transparency and reliability of water trading. During the feasibility study, Civic Ledger developed a peer-to-peer trading platform that provided irrigators with secure and transparent trading.

Another approach is presented by Pincheira et al. [97] aiming to streamline energy costs through good management of water resources, integrating IoT sensors and blockchain. The architecture includes IoT devices, a public blockchain and smart contracts representing the interests of different stakeholders in water management. Moreover, smart contracts have the role of regulating the distribution of incentives among farmers.

Grigoras et al. [98] use the blockchain platform for trading, thus reducing energy costs. Another approach presented by Rottondi and Verticale [99] proposes a solution based on gamification, through which data are taken from IoT devices, which come from the utility company. Furthermore, data are used in the gamification process, and blockchain plays an essential role in preventing dishonest behavior.

A recent study detailed in [87] presents an intelligent system through which irrigation communities can communicate and manage water resources. Community members use blockchain to increase confidence in the data collected from IoT devices.

However, in order to achieve an optimal system from the point of view of water resources management, we must consider several aspects, including energy efficiency and low cost, in order to ensure cost-effective and long-term operations [100]. Hyperledger Fabric was used by Wang et al. [101] in the context of a crowdsourcing framework for energy generation and trading, using a marginal amount of energy for consensus when compared to other blockchains.

When considering the integration of blockchain in MCS, Yang et al. [102] proposed a blockchain solution, addressing the problem of location privacy in crowdsensing frameworks by using private blockchains.

The overall effect on platform utility is evaluated by Kadadha et al. [103] with SenseChain, a decentralized crowdsensing framework built on Ethereum blockchain, which is compared to a centralized framework, i.e., greedy selection. The simulation results show an increase in both the level of participation and the quality of the sensing data. Similar conclusions are presented by Jia et al. [104], who propose a blockchain-based incentive mechanism with an emphasis on location privacy protection. The MCS platform was used for collecting noise data in a campus environment, while the participation rate increased from $20 \%$ to $80 \%$ by using the proposed incentive mechanism when compared to traditional MCS. 


\section{Survey}

In this section, we describe an in-depth analysis we performed of the current state of the gaming industry and scientific papers with related topics. The main objective of this data-driven survey was to highlight the trends in the industry and to provide a real-world background for our proposed solution.

A data-driven survey of video game scores on Metacritic is presented by Predescu and Mocanu [105], focusing on PC games and a classification by genres. The PC has always been a popular gaming platform, but a considerable number of game consoles created a rich variety of options for the increased interest in video games among the younger generations, from handheld devices (e.g., Game Boy, Nintendo DS, Sony PlayStation Portable) to home consoles (e.g., Sony PlayStation/2/3/4, Nintendo Wii, Microsoft Xbox/360/One) [106].

Mobile games are different from the perspective of distribution, integrated user rating and overall experience. The mobile ecosystem provides a common framework for application distribution which includes mobile games, that are subject to similar policies, requirements and design patterns.

\subsection{PC Games}

To evaluate the trend in the PC gaming industry, the average user ratings were extracted from Metacritic for a number of 4601 titles. The evolution of PC games from the perspective of user scores is shown in Figure 2a, while the number of new releases per year is shown in Figure $2 b$.

The data were extracted from the web pages in the category "Best Games of All Time". While older games have not been documented in terms of user reviews until the internet age, since the introduction of game-related websites, the data show decreasing interest in new games, according to the user scores, while the number of new releases has been increasing over time.

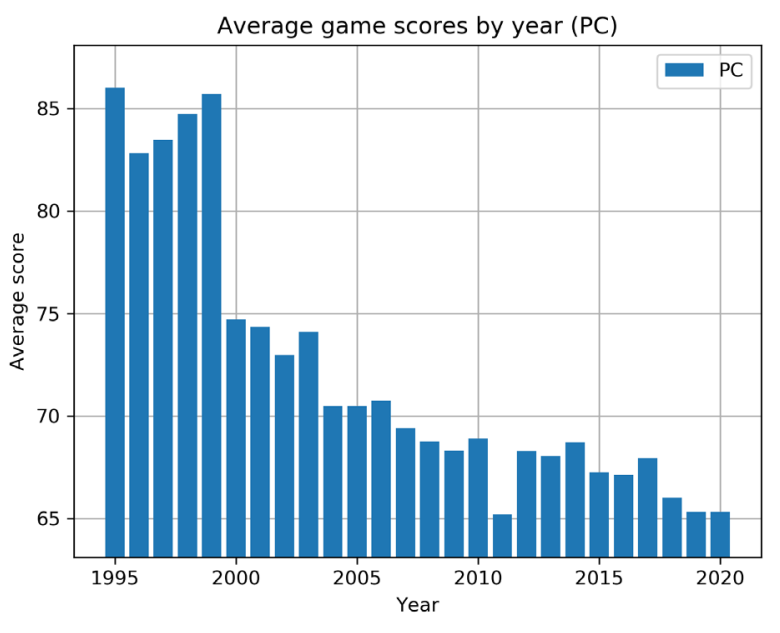

(a)

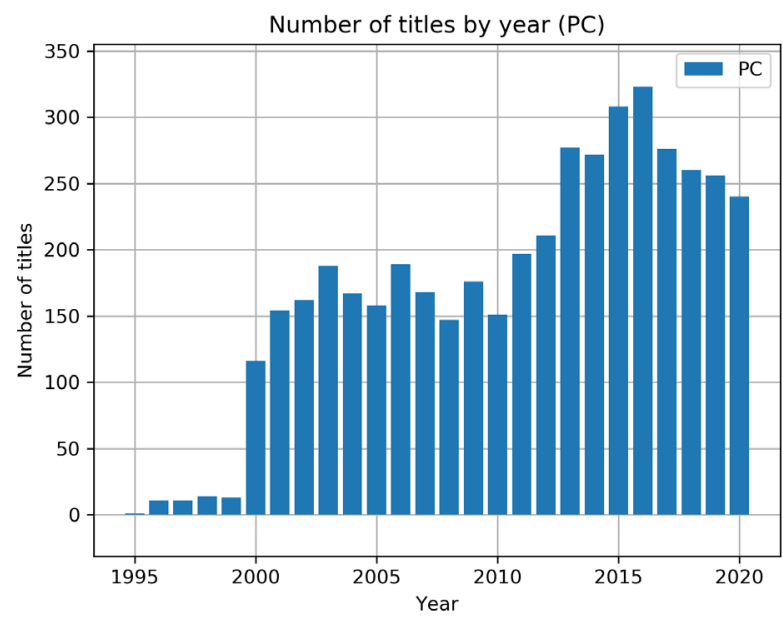

(b)

Figure 2. Evaluation of Personal Computer (PC) games statistics over time; (a) average user scores by year; (b) number of new releases by year.

\subsection{Console Games}

For console games, the data were extracted from Metacritic for each console type, accounting for a number of 9569 titles. The games were extracted from the category "Best of" for each console (e.g., "Best of PS3") and then combined to show an overview of the entire industry.

The evolution of console games from the perspective of user scores is shown in Figure 3a, while the number of new releases per year is shown in Figure 3b. When compared to PC games, the results are similar, considering more modern consoles in the 
evaluation (PlayStation 2/3/4, Wii, Xbox, Xbox 360, Xbox One, Nintendo DS), where user data are available.

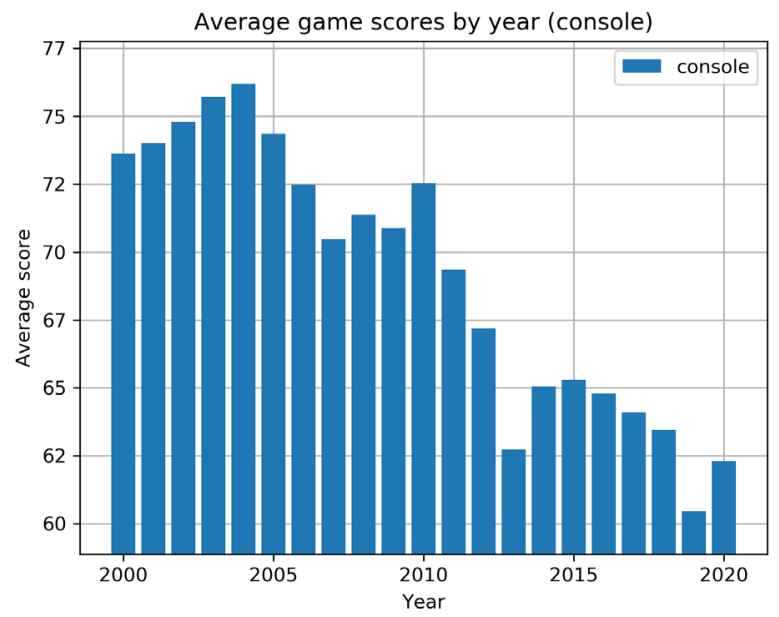

(a)

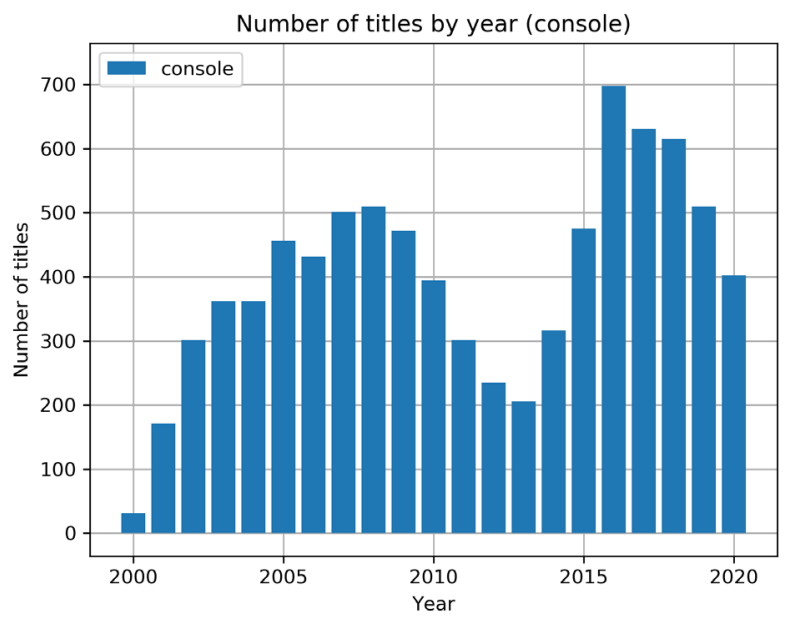

(b)

Figure 3. Evaluation of console games statistics over time; (a) average user scores by year; (b) number of new releases by year.

In contrast to PC games, where hardware can be upgraded to achieve improved performance and visuals, the console type can play an important role in the overall gaming experience. Moreover, the game interaction is different on different consoles, depending on the physical controls and surroundings. Therefore, the results by console type are shown in Figure $4 \mathrm{a}$ for the average user score and in Figure $4 \mathrm{~b}$ for the number of new releases by year.

In general, the scores are similar across different consoles. However, there are some games that are exclusive to a particular console (e.g., Nintendo Wii), which, linked to a higher degree of novelty, can translate into higher user scores (e.g., 2012-2014).

Another difference when compared to PC games is revealed in Figure $4 \mathrm{~b}$. As the industry progresses, new consoles are released that are not usually backward compatible with older hardware, while games are rarely ported for previous generations of consoles.

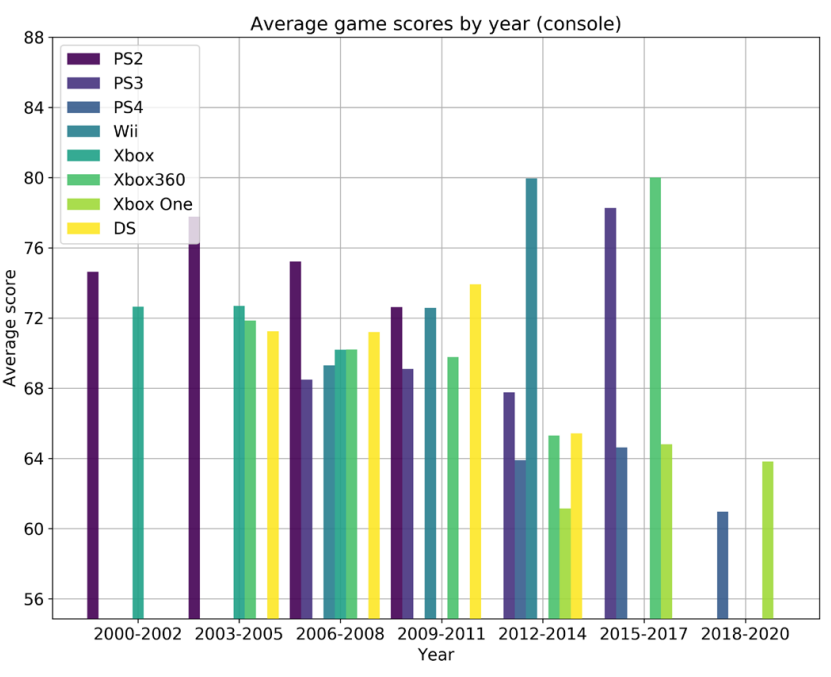

(a)

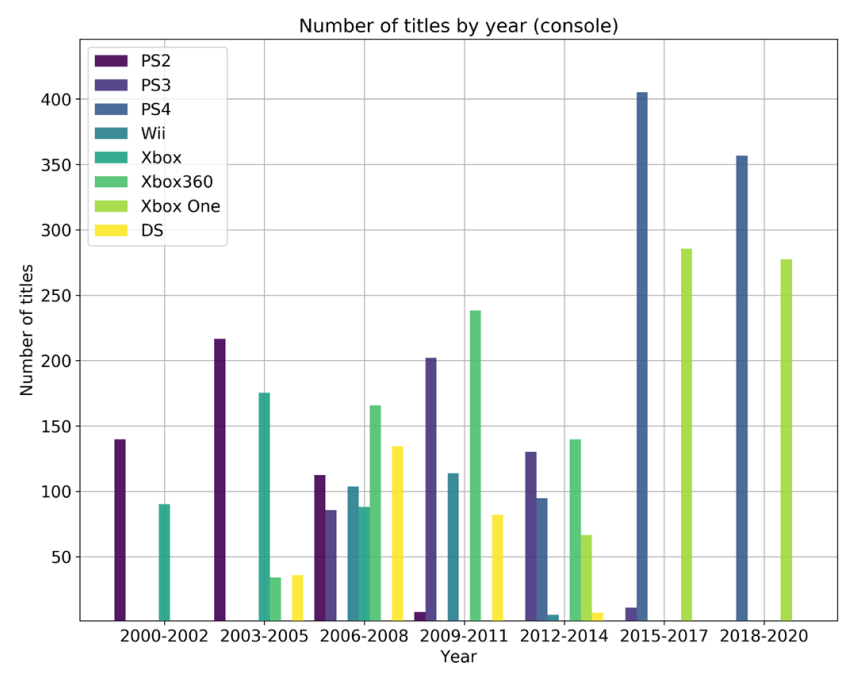

(b)

Figure 4. Evaluation of console games statistics over time by console type: (a) average user scores by year and console type; (b) number of new releases by year and console type. 


\subsection{Mobile Games}

Extracting data about mobile games on Play Store presents a different set of challenges: (1) there is no way to list all games from the store; (2) there are multiple categories that only provide the most relevant results and suggestions; (3) to find a specific game, the application ID must be known in advance.

There is, however, an accurate, crowdsourced video game database covering a broad range of platforms, including mobile (Android, iOS). Therefore, the list of all registered Android games from 2008 to 2020 was extracted from MobyGames [107]. However, user rating was not available on the platform, and each title was searched on Google to find the application ID and then to be able to extract the data from the store. A total of 7886 games were listed on the website, of which 6086 were found on Play Store.

The results are presented in Figure $5 \mathrm{a}$, showing the average user ratings by year, while the number of new games by year is shown in Figure 5b. The mobile industry has seen a major development since the introduction of the smartphone, which translates into an increasing number of mobile games until 2016. As the mobile application market reached maturity and new policies were introduced, there has been a decrease in this sense. However, with the exception of 2008, where some of the first few games were developed for the platform, the average user scores have been steadily increasing, from 4.02 stars (i.e., $80.4 \%$ ) in 2009 to 4.18 stars (i.e., $83.6 \%$ ) in 2020, showing a high overall interest in mobile games when compared to PC and console games.

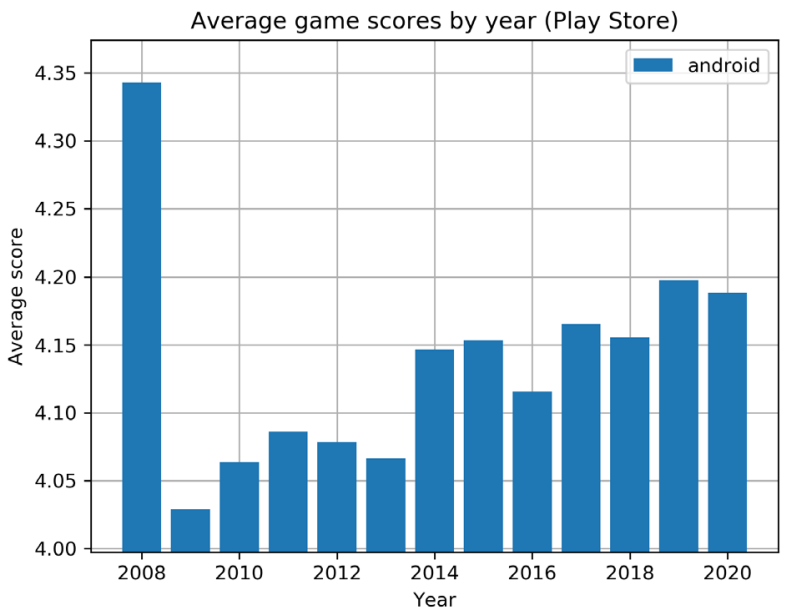

(a)

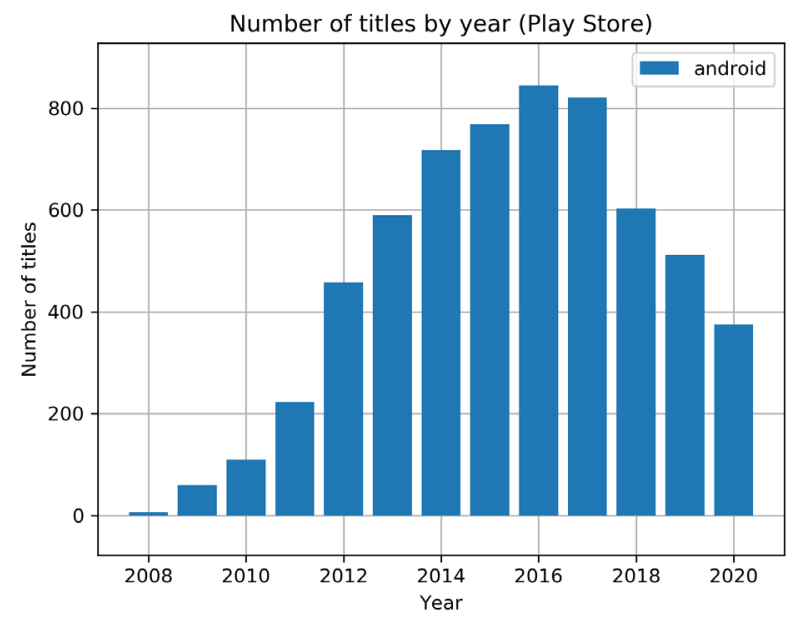

(b)

Figure 5. Evaluation of mobile games statistics over time; (a) average user rating by year; (b) number of titles by year.

\subsection{Related Papers}

To provide a background of recent academic research in related fields, a keyword search was performed on Google Scholar. Research papers were extracted, with titles containing the keywords (Blockchain, Crowdsensing, Serious Gaming, Crowdsensing + Blockchain, Urban Water, Blockchain + Water, Crowdsensing + Water). Therefore, the combination of keywords related to different technologies and concepts was also analyzed from the perspective of existing works in the literature to evaluate the level of interest and novelty. The search was limited to 50 pages for each category, while the search engine returned at most 10 results for each page, showing the most relevant results first (i.e., sort by relevance).

In Figure $6 a$, the number of papers by year is shown for each search term, of which Blockchain and Crowdsensing were found to be most popular in 2018, while Serious Gaming shows a rather constant trend. From the evaluated combinations, Crowdsensing+Blockchain, and Blockchain + Water revealed a number of papers since 2018, showing an interesting link. 
The distribution of keywords is shown in Figure 6b, where the term Crowdsensing is revealed as the most commonly found in publication titles, followed by Blockchain, Urban Water and Serious Gaming. While the results are based on publication titles, other associations may be revealed when comparing the abstracts, which is a subject of further research.

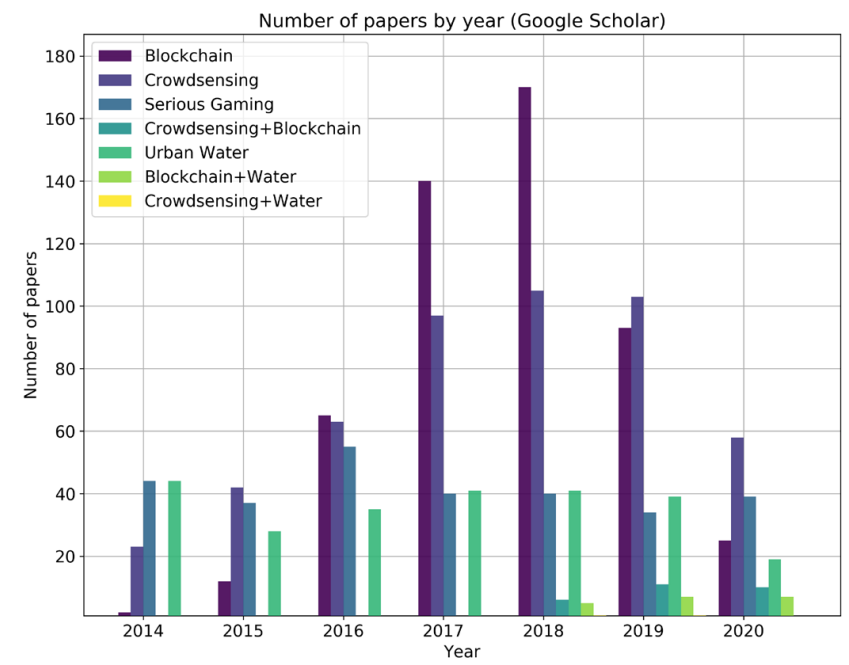

(a)

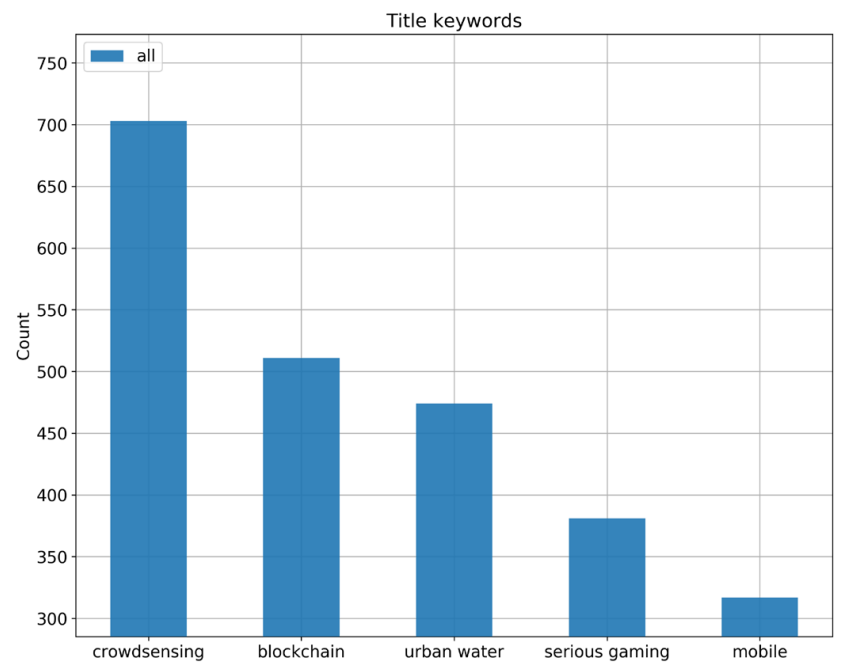

(b)

Figure 6. Evaluation of research papers statistics over time by keywords in the title; (a) number of papers by year and keywords; (b) histogram of paper keywords.

\section{Methodology}

In this Section, the algorithms and methods are described for the SG-based crowdsensing solution in Section 4.1 and the blockchain data model in Section 4.2, to provide a background for the solution proposed in Section 5 .

\subsection{Crowdsensing Model}

We considered the crowdsensing solution as a two-stage routing problem. The allocation of tasks is translated into a Vehicle Routing Problem (VRP) with capacity constraints, while the incentive mechanism is further refined using a Vickrey-Clarke-Groves (VCG) auction model for truthful reporting.

\subsubsection{VRP-Based Task Allocation Model}

The VRP model represents the platform-centric approach for MCS evaluated in this paper. While there are multiple variations of VRP used for solving different problems, such as those presented in Section 2, the model was adapted to the MCS scenario proposed in Section 5.1 by defining the context and required constraints.

The VRP-based task allocation model is defined as follows: players are represented by vehicles, with capacities according to the reputation levels, while distance constraints define, e.g., the maximum travel distance. Tasks are represented by customers, with fixed locations and demands set according to the assigned priorities. Each vehicle has a starting point, i.e., player home, which corresponds to a depot, while the other nodes are associated with customers.

To define the mathematical model in the context of the proposed solution, we consider the following notations: 


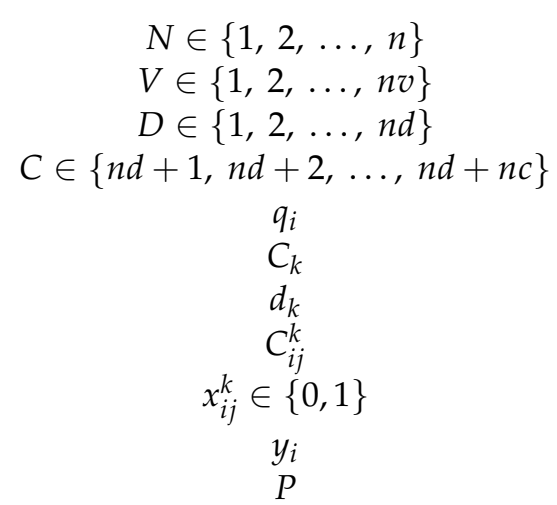

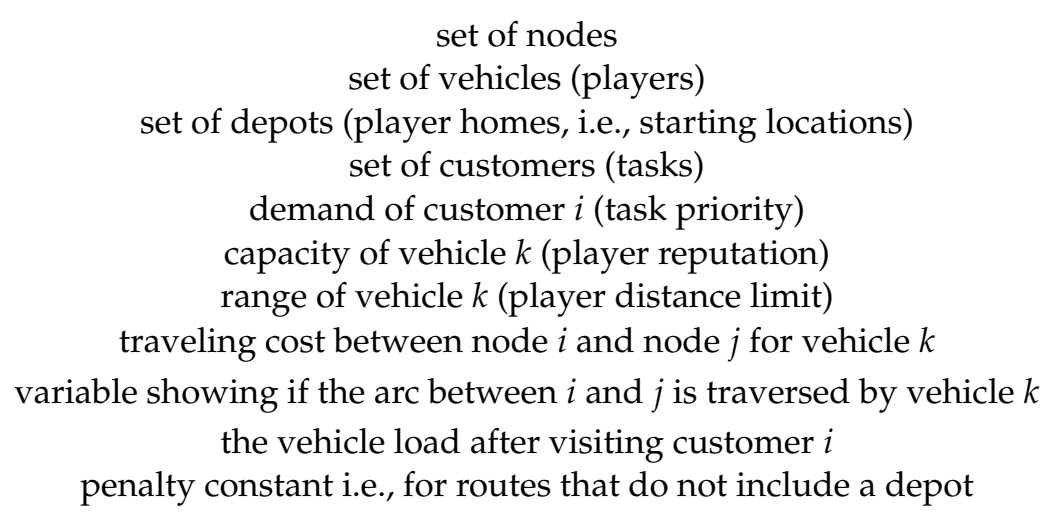

set of nodes

set of vehicles (players)

set of depots (player homes, i.e., starting locations)

demand of customer $i$ (task priority)

capacity of vehicle $k$ (player reputation)

The vehicle routing problem (distance-constrained multi-depot vehicle routing problem with heterogeneous fleet capacity) can be defined by a combinatorial optimization and integer programming problem [108-111], with an objective function defined in Equation (2) that consists in minimizing the total cost, subject to a set of constraints that are presented below:

$$
\min _{x} \sum_{i=1}^{n d+n c} \sum_{j=1}^{n d+n c} \sum_{k=1}^{n v} c_{i j}^{k} x_{i j}^{k}
$$

Constraints in Equations (3) and (4) are imposed to ensure that each customer (task) is visited (reported/resolved) exactly once by a vehicle (player) and then the vehicle should proceed to the next customer (task).

$$
\begin{aligned}
& \sum_{i=1}^{n d+n c} \sum_{k=1}^{n v} x_{i j}^{k}=1, \forall j \in C \\
& \sum_{j=1}^{n d+n c} \sum_{k=1}^{n v} x_{i j}^{k}=1, \forall i \in C
\end{aligned}
$$

Constraints in Equations (5) and (6) state that each vehicle (player) must start from a depot (player home) and return to the same depot after completing the tour, while it is not required to dispatch all vehicles.

$$
\begin{aligned}
& \sum_{j=n d+1}^{n d+n c} x_{i j}^{k} \leq 1, \forall k \in V_{i}, i \in D \\
& \sum_{i=n d+1}^{n d+n c} x_{i j}^{k} \leq 1, \forall k \in V_{j}, j \in D
\end{aligned}
$$

Constraints in Equations (7) and (8) enforce the condition that each vehicle (player) must belong to a depot (player home).

$$
\begin{aligned}
& \sum_{j=n d+1}^{n d+n c} x_{i j}^{k}=0, \forall k \notin V_{i}, i \in D \\
& \sum_{i=n d+1}^{n d+n c} x_{i j}^{k}=0, \forall k \notin V_{j}, j \in D
\end{aligned}
$$

The route continuity constraint, i.e., flow conservation equation, is defined in Equation (9).

$$
\sum_{i=1}^{n d+n c} x_{i j}^{k}=\sum_{i=1}^{n d+n c} x_{j i}^{k}, \forall j \in C, k \in V
$$


The maximum capacity constraint (player reputation) is defined in Equation (10), while the maximum range constraint (travel distance) is defined in Equation (11) for each vehicle (player).

$$
\begin{aligned}
& \sum_{i=n d+1}^{n d+n c} \sum_{j=1}^{n d+n c} q_{i} x_{i j}^{k} \leq C_{k}, \forall k \in V \\
& \sum_{i=n d+1}^{n d+n c} \sum_{j=1}^{n d+n c} d_{i j} x_{i j}^{k} \leq d_{k}, \forall k \in V
\end{aligned}
$$

In Equation (12), the constraint further imposes route continuity and prohibits the formation of routes that do not include a depot (player home).

$$
y_{i}^{k}+d_{j} x_{i j}^{k}-y_{j}^{k} \leq P\left(1-x_{i j}^{k}\right), \forall k \in V, i \in N, j \in C
$$

In Equation (13), the constraint requires that there is no inter-depot route, i.e., route that includes other depots (player homes).

$$
x_{i j}^{k}=0, \forall k \in V, i, j \in D
$$

\subsubsection{VCG-Based Auction Model for Truthful Reporting}

The VCG-based user-centric approach is defined in Section 5.1 in the context of the proposed MCS platform. In VCG auction, there are multiple items and agents, with each agent placing a bid for each item without knowing the valuations of others. The auction system assigns the items according to their true valuations, while each agent pays only the marginal value when compared to the other agents, encouraging truthful bidding.

The Vickrey-Clarke-Groves mechanism $[112,113]$ is defined by the following optimization problem (Equation (14)) that maximizes the total utility, based on the optimal allocation of items to agents:

$$
X(\hat{v})=\arg \max _{x \in X} \sum_{i=1}^{n} \hat{v}_{i}(x)
$$

where $\hat{v}_{i}: X \rightarrow R_{+}$represents the valuation (bid) of agent $i$ with regards to each possible outcome, i.e., $x \in X$.

The payment function for agent $i$ depends on the valuations of other agents for a given outcome, i.e., $x \in X(\hat{v})$, with the agent $i$ and $x \in X\left(\hat{v}_{-i}\right)$, without the agent $i$, as defined in Equation (15):

$$
p_{i}(\hat{v})=\sum_{j \neq i} \hat{v}_{j}\left(X\left(\hat{v}_{-i}\right)\right)-\sum_{j \neq i} \hat{v}_{j}(X(\hat{v}))
$$

The mechanism promotes truthful bidding through incentive compatibility (truthful bidding is a dominant strategy), individual rationality (each truthful agent receives a payment) and efficiency (truthful bidding maximizes social welfare).

\subsection{Blockchain Model}

We considered the blockchain as a solution for storing the collected data because of its transparency, security and accessibility. To demonstrate these properties, we look deeper at how this architecture works, what the key components are and how trust between peers is guaranteed. It is known that the blockchain architecture brings multiple benefits, but it must be noted whether its integration into the proposed solution improves performance or not.

Hyperledger is one of the newest solutions in the blockchain world and came with a new vision in the way of developing blockchain solutions [114]. Each Hyperledger project/solution respects a predefined architecture and is composed by the following components: Consensus Layer, Smart Contract Layer, Communication Layer, Data Store Abstraction, Crypto Abstraction, Identity Services, Policy Services, APIs and Interoperation [115]. 
To reach consensus, Hyperledger Fabric uses the Raft algorithm [116] as shown in Figure 7. It resembles a voting system, in which the nodes play various roles (followers, candidates, leaders), depending on their state. Specifically, each node plays the role of the candidate and tries to convince the other members to vote for him. He becomes a leader if more than half of the voters/nodes support him. Therefore, any node in the algorithm can be a candidate and can send requests to other followers. Moreover, if more than half of the followers send an answer, then the elections are successful, and the candidate with the most votes becomes the leader.

In addition, a log containing the entire election history is copied to the followers. If the new leader goes down, a new leader will be chosen, according to the steps saved in the log.

Another feature of the nodes is the measurement of the heartbeat through a timer. Normally, leaders have priority in measuring their heartbeat. If they no longer respond properly, then the leader is considered lost because he is no longer able to provide services. Therefore, he becomes a candidate and participates in the election of a new leader for a new term.

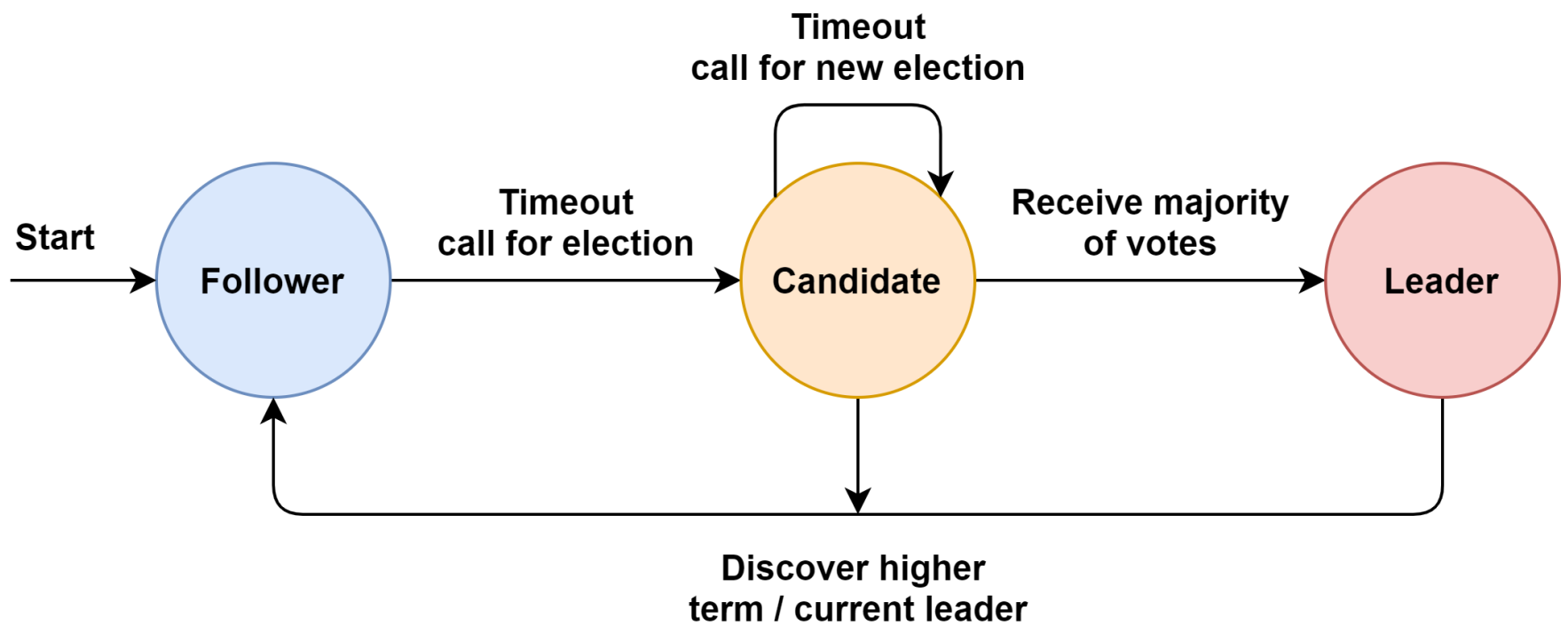

Figure 7. Raft algorithm.

The advantage of using the Raft algorithm is the possibility of losing nodes, including leader nodes, without affecting the system as long as there is a majority of nodes that can take command. Therefore, it can be stated that the Raft algorithm is crash-fault-tolerant (CFT). For example, if a network contains three nodes, then one node can be lost, leaving another two nodes functional; if the network has five nodes, then two nodes can be lost and the other three can function without problems. Therefore, as there are more nodes in the network, the number of lost nodes does not influence the proper functioning of the system. In other words, this feature offered by Raft ensures the availability of services and offers a high degree of security [117].

\section{Proposed Solution}

The proposed solution combines the crowdsensing model and SG platform with a blockchain-enhanced data model to achieve increased platform utility for crowdsensing in urban water infrastructure.

\subsection{Crowdsensing Model}

For georeferenced serious games where a set of challenges are distributed on the map, the game design for achieving optimal distribution of players to complete the tasks can be defined as a dispatch optimization problem combined with a reward-based incentive 
mechanism. The tasks involve issue reporting in the context of urban water and can be extended for many use cases.

The dispatch optimization problem can be formulated as an application of the Vehicle Routing Problem (VRP) as shown in Figure 8 , where the players, i.e., $\left(P_{1}-P_{3}\right)$ are modeled as vehicles and tasks, i.e., $\left(T_{1}-T_{10}\right)$, are modeled as customers, with the objective of cost optimization (e.g., distance traveled) under specific constraints, as described in Section 4.1.

Therefore, geolocation and other constraints can serve as a filter for dispatching tasks to players to achieve a cost-effective routing strategy for increased platform utility in terms of truthful reporting and quality of sensing data.

However, to achieve an overall platform utility, the reward-driven incentive must be counterbalanced by a regulation strategy. The reputation score is the main currency in the game and must be defined in order to achieve optimal incentives for crowdsensing tasks while reducing the risk of overreporting or false reporting.

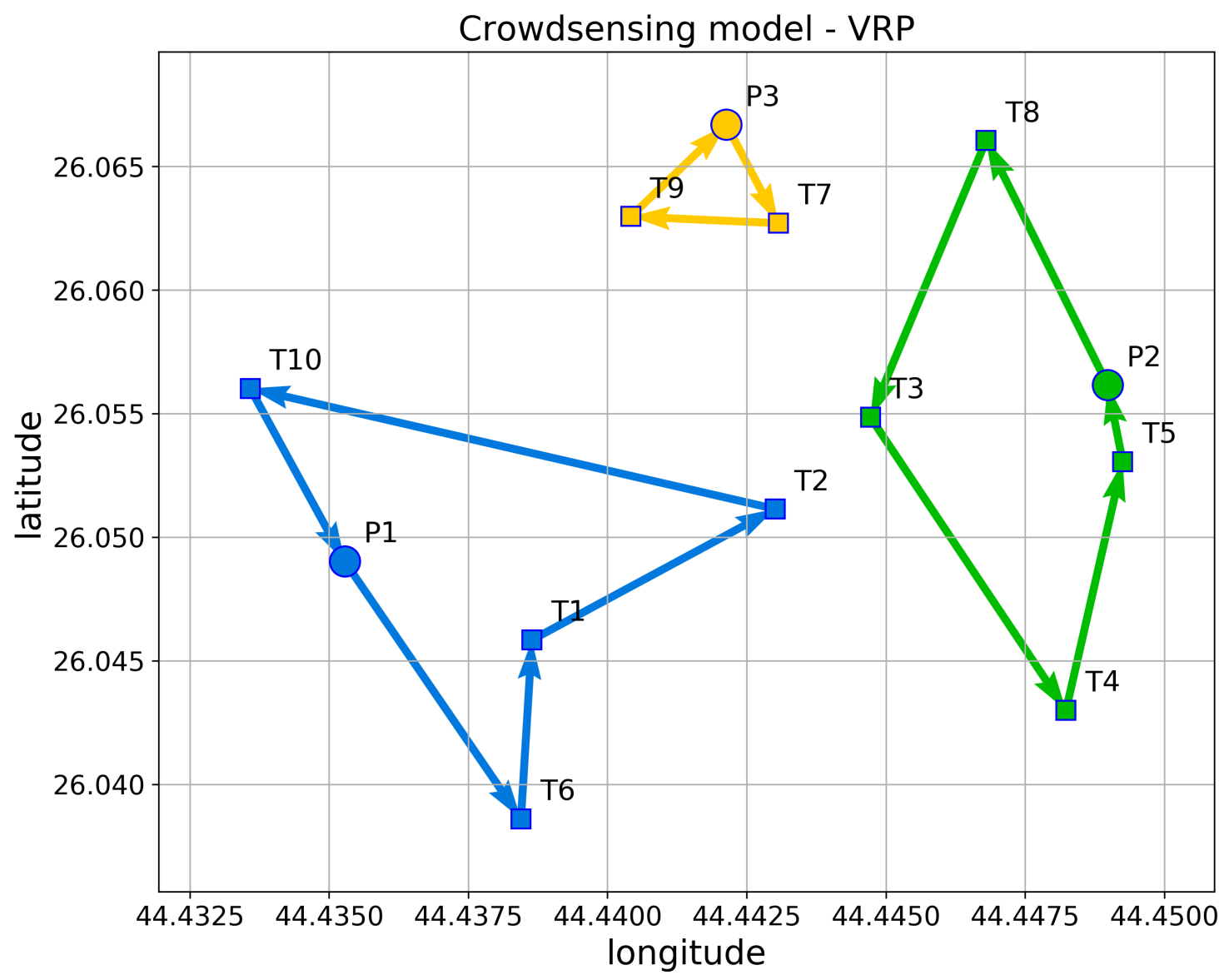

Figure 8. Crowdsensing model with Vehicle Routing Problem (VRP) solution.

In this sense, the VRP can be defined with the capacity constraints for each player according to the reputation. At each iteration of the optimization algorithm, the capacities are updated. In the case of truthful reporting, the capacity is increased according to the reward, which allows for routing towards the next tasks. We considered three types of players: 100\% match (truthful and accurate reporting), $80 \%$ match (truthful and mostly accurate reporting) and $50 \%$ match (false/inaccurate reporting).

To evaluate the model and different types of behavior, we run the optimization problem on the entire range of distributions in terms of geographical coordinates of the players. We considered fixed coordinates for the tasks and variable starting coordinates for players to evaluate the average cost of the system (total distance traveled) for each player 
behavior and therefore to assess the platform utility for encouraging truthful participation. The results are presented in Section 7.1.

To translate the problem formulation into VRP constraints, we considered the following: (1) player reputation is represented by vehicle capacity; (2) player effort is represented by vehicle fuel level; (3) task priority is represented by customer demand.

Therefore, an effective routing strategy that stimulates truthful reporting through reputation incentives accounts for the reputation of players that report the issue as well as players that validate the issue, allowing for an overall regulation of the system towards improved platform utility.

The proposed framework allows for the evaluation of reward strategies based on the estimated level of trust/reputation of players. The rewards can be defined based on the required capacities that satisfy a given distribution of issues.

To achieve the optimal solution in terms of the assignment of the most truthful players to a higher number of priority issues, a balance must be achieved between the relative priorities and the reputation of players. An optimization problem can be defined when comparing the results for each player type with the relative task priority adjusted by a reward factor to achieve an optimal allocation of tasks.

The VCG auction model can be used to provide incentives according to the player type and estimated cost. Therefore, the players submit their bids according to the expected cost for each task and their own reputation level. The algorithm provides the optimal assignments and rewards according to the auction model, which incentivizes truthful bidding.

The same methodology and conditions were used to simulate the VCG-based allocation of tasks, as described for VRP, and the results are presented in Section 7.1.

\subsection{Blockchain Framework}

For the development of the proposed solution, the blockchain component is based on a Hyperledger Fabric implementation, which presents key features for an efficient implementation in the context of the proposed solution.

Some of the key advantages of this technology are given by its open-source nature, keeping the transaction history in a data structure, and not requiring the use of cryptocurrencies. The transaction proposals are sent to Endorsing Peers, i.e., the nodes that approve the verification of transactions and generate a response that contains the read-and-write set. Moreover, the output is sent to the node that initiated the transaction, which receives the output from all Endorsing Peers and sends it to the Orderer node. In the next stage, the Orderer node observes all the transactions and forms a block with them in ascending order. The newly formed block is sent to all committer nodes, which add a block to their own register.

Based on a smart contract and following the request made by the user, the data can be queried or added to the blockchain. Moreover, the data can be accessed using a REST API.

The proposed architecture, described in the Figure 9, uses Docker to generate the containers for the Orderer node, Peer nodes and CouchDB (non-relational database) with a REST API for the different user types defined in Section 5.3, representing different stakeholders in the proposed solution.

Each peer container has its own registry saved in CouchDB. To ensure trust between peers, certification authorities were used for each peer. Moreover, a separate certification authority was used for the Orderer.

To provide data consistency and increased security, the data register was replicated on multiple nodes. An essential feature of the integration of blockchain technology in the proposed solution is audit support, as described in previous research [118]. Given that data in the blockchain cannot be modified or deleted, it is a useful tool by which the data from the database can be compared to the data stored in the blockchain, providing an efficient solution for the trust problem in MCS. 


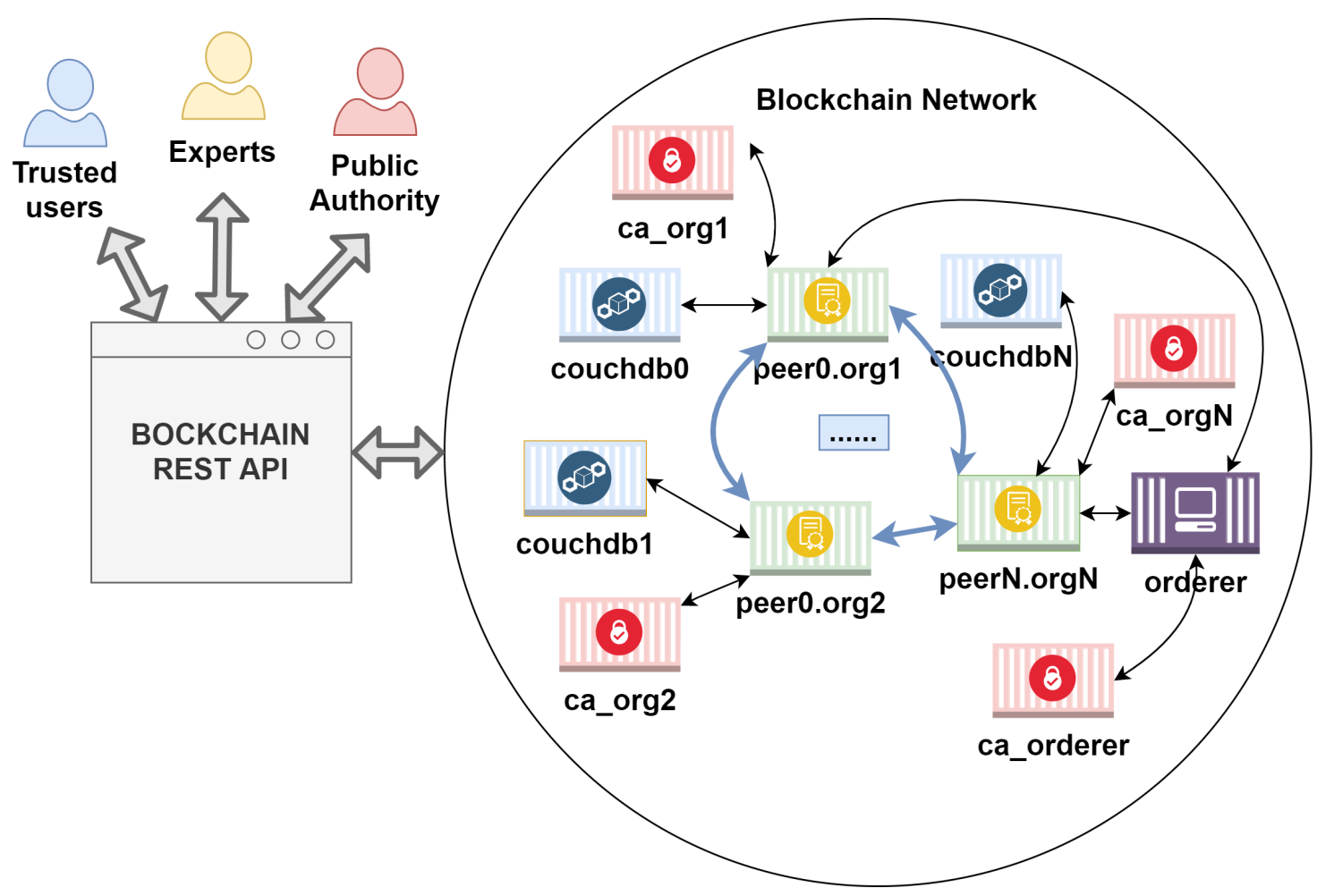

Figure 9. Blockchain architecture.

\subsection{Serious Gaming Platform}

The serious game is defined based on a set of roles assigned to players for the crowdsensing tasks as shown in Figure 10. Issue discovery is delegated to the Blue Team, which consists of crowdsourced opportunistic sensing and event (E) reporting. The reports are published on the map (FIND) and visible to the other players.

The Yellow Team is designated for solving the reported issues (FIX) and consists of field experts that are available nearby. The reports can be either confirmed or invalidated by the Yellow Team, while the proof of validation is uploaded on the platform. Upon validation, the Blue Team member receives a reward for truthful reporting.

The Red Team represents the public authorities, with trusted experts monitor and that validate (VALID) the reports. Upon solving an issue, the reward is assigned to the Yellow Team member. If the issue is evaluated as false reporting by the Red Team, both the corresponding rewards to the Yellow Team and the Blue Team are invalidated. In this sense, for the Blue Team, the Yellow Team can assign rewards for truthful reporting, while the Red Team can invalidate them based on expert reviews.

The rewards have a direct influence on player reputation, while truthful reporting is enforced by the crowdsensing model.

The SG platform is defined by a mobile application for crowdsensing in urban water infrastructure, based on the urban exploration platform developed with Leplace Global [119]. The players/citizens can report issues with regard to water utilities and place them on the World Map. Gamification is used to increase the level of participation and interaction with the environment, while responsive design and visual aspect provides for an attractive game environment with real-world implications. The main view is shown in Figure 11, with the location of issues and players according to the experimental setup used in this paper. 


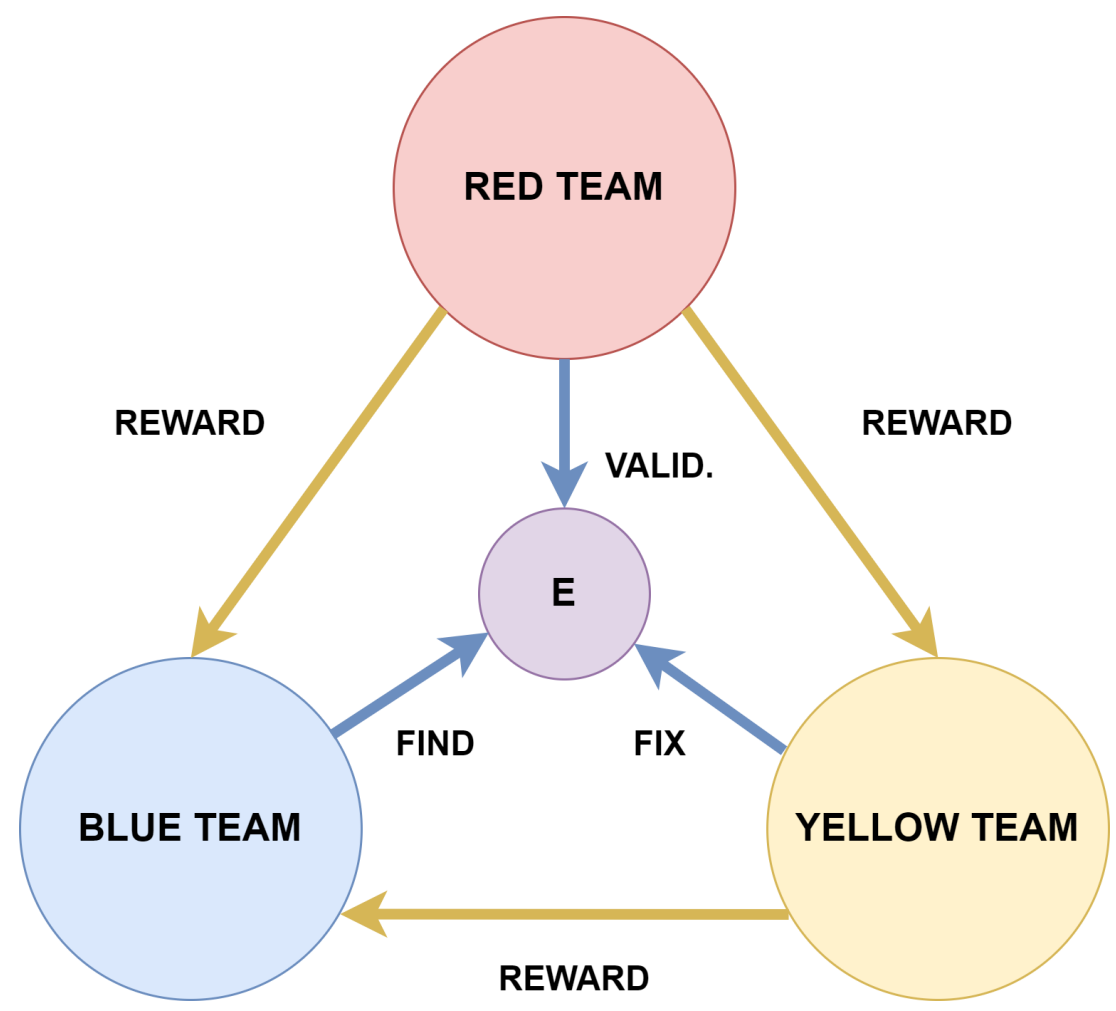

Figure 10. Serious game model.

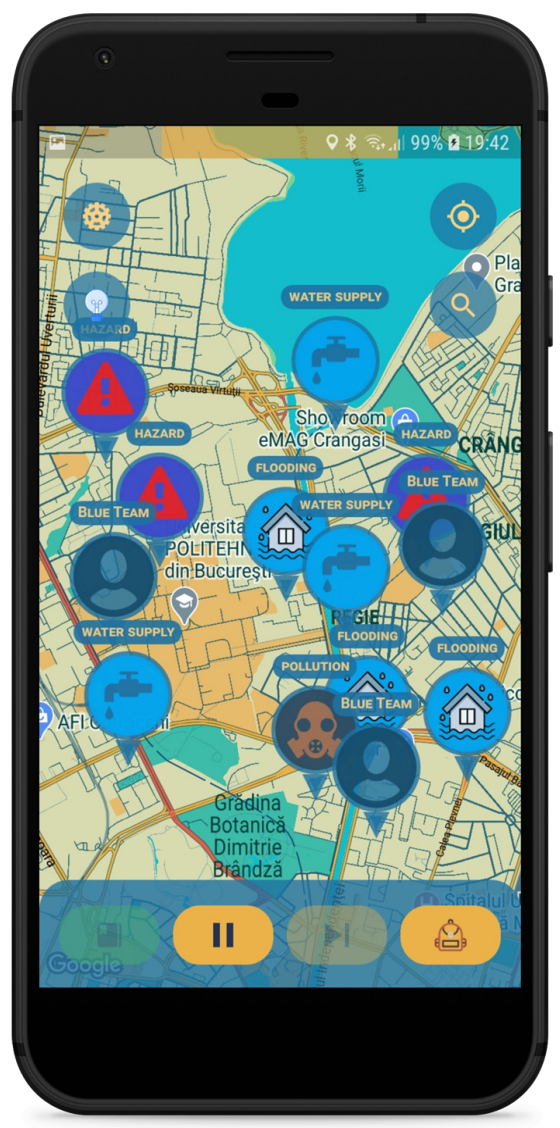

Figure 11. Watergame mobile application prototype. 
While there are different kinds of issues that can be found in urban water infrastructure, the map view reveals nearby issues as reported by the players: flooding, water supply (e.g., leaks or service interruptions), pollution and hazards (other circumstances). The player locations are shown on the map for experimental purposes, while in real-world applications, collecting real-time location data may be prohibited by privacy laws according to the use case.

The crowdsensing model presented in Sections 4.1 and 5.1 can be used to define the allocation of tasks for each player and to provide the incentive mechanism. Reported issues are registered on the blockchain as described in Sections 4.2 and 5.2, for an increased level of trust for the stakeholders, through secure data storage and transparency.

The proposed components fill the existing gaps in mobile crowdsensing applications by addressing the motivation, trust and coverage issues from three different perspectives. Therefore, the overall platform utility, i.e., coverage and sensing cost, is influenced by: (1) the crowdsensing model as the base for the in-game economy; (2) an attractive game design to increase the level of participation; (3) the perceived level of trust, ensured by the decentralized storage ledger.

\section{Materials and Methods}

The VRP-based solution is implemented in Python using Google OR-Tools [120], which allows for modeling the route optimization problem in a general format, with the required constraints that can be added, of which we used: capacity constraints, multiple depots and distance constraints.

While the Google Directions API [121] can be used to compute the distance matrix for a given set of points on the map, the platform allows for a limited number of requests within a given budget. Therefore, the experimental solution includes an offline calculation module for simulating random player locations.

The VCG-based solution is implemented in Python using a VCG auction implementation [122] and integrated into the simulator to evaluate multiple scenarios in terms of optimal task allocation with regards to a reputation-based bidding scheme.

The crowdsensing model simulator is available on GitHub [123] and evaluates the VRP and VCG models for different configurations in terms of player distributions on the map.

The blockchain performance evaluation framework is available on GitHub as well [124] and is based on the Hyperledger Caliper benchmark tool [125]. The original repository contains the required configurations for evaluating multiple blockchain architectures, while we focused on the proposed scenario for validating the Hyperledger Fabric performance.

\section{Results}

To provide an overview of the proposed solution in terms of performance and accuracy, the crowdsensing model is evaluated in Section 7.1, and the blockchain model is evaluated in Section 7.2.

\subsection{Crowdsensing}

The crowdsensing solution is evaluated using the two proposed formulations based on the VRP model and the VCG auction model, each providing different and complementary perspectives on the player-task allocation problem, i.e., platform-centric/user-centric.

\subsubsection{VRP Model}

The optimization model is implemented in Python, based on Google OR-Tools framework, which provides a general formulation of the VRP with multiple layouts and constraints. To evaluate the VRP load balancing, i.e., task allocation, we considered a set of 10 locations in Bucharest, and three players that correspond to the simulated behavior: accurate reporting, mostly accurate reporting and false reporting. The routing module is initialized with the distance matrix, number of players and location of depots (starting locations). 
The selection of optimal routes is based on a distance function and the defined consumer demands, while vehicle capacities (player reputation) and fuel level (travel distance) are modeled as capacity constraint dimensions. A penalty function is added, allowing to exclude nodes in order to solve the optimization problem, whereas no feasible solution could be found within the given conditions (e.g., total consumer demand exceeds the capacity of the fleet). The optimization algorithm solves for optimal cost within the provided constraints and time limit, returning the optimal path for each vehicle, which provides the initial assignment of tasks to nearby players.

To evaluate the effect on the distribution of tasks according to the player reputation, we defined the capacities in the context of VRP as follows: accurate reporting (100), mostly accurate reporting (80) and false reporting (50). Then, we proceed to evaluate how the relative importance of the issues found in the locations scattered across the city should be related to the player reputation to achieve optimal load balancing. We considered a fixed distribution of associated demands and a reward factor that is applied for each demand.

For the experiment, we evaluated the effect of this reward factor in the range of 1 to 15 , where a factor of 5 results in a balanced overall distribution (total rewards vs. total reputation). As the starting location of players may affect the accuracy of the results, we considered a random initialization using a bounding circle. The VRP is evaluated for 1000 iterations, and the average load (resource allocation) is shown in Figure 12a for each player type.

In this evaluation, the optimal reward factor should be 5 or greater, as there is an increase in standard deviation for lower values, as shown in Figure 12b, which accounts for less predictable results. In fact, for a reward factor of 1, the optimal allocation of tasks is not satisfied, whereas a factor of 5 or greater results in more predictable distributions while the proposed objective is achieved.

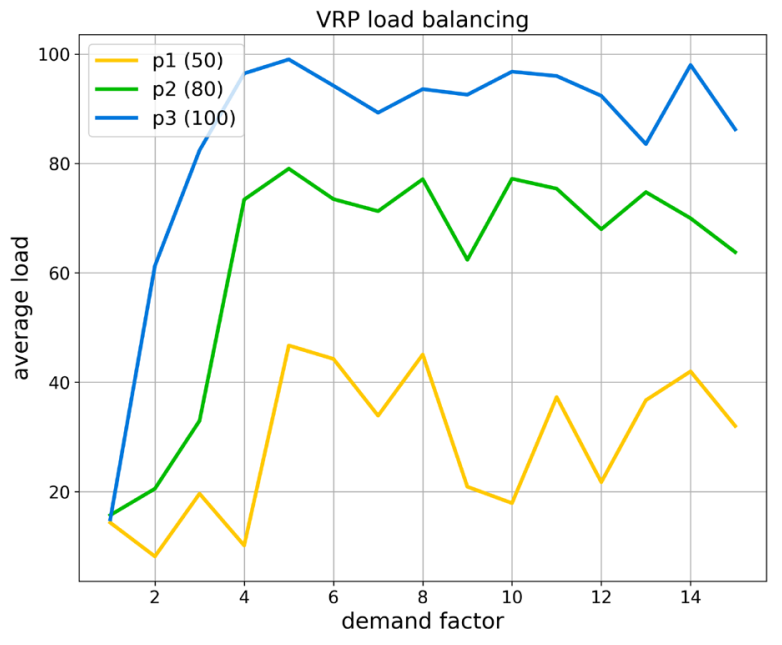

(a)

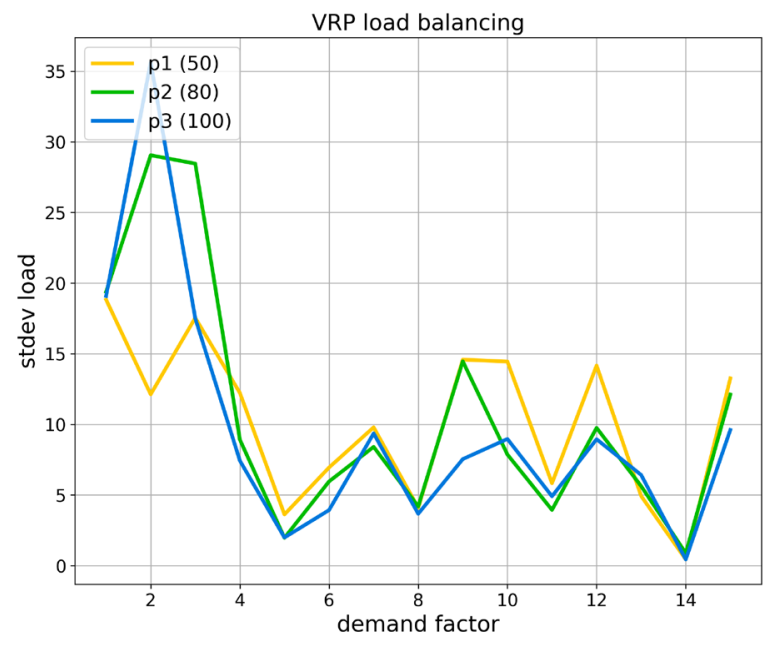

(b)

Figure 12. VRP load balancing; (a) average load; (b) standard deviation.

According to the results, while the optimization problem is solved either way, the optimal reward factor should be defined to achieve the desired outcome for the incentive mechanism. Therefore, there should be a balance between the reputation of players and the rewards allocated for each available sensing task.

\subsubsection{VCG Model}

The VCG auction model is based on a Python implementation that receives the input data from a text file describing the available items and bids for each participant and returns the allocation of items according to the truthful bidding strategy. The distance matrix is translated into the input format as follows: the bids for each task are defined as a function 
of distance (reported cost) and normalized player reputation $R_{i} \in[0,1]$, with the higher reputation of players accounting for more truthful bidding. The total payoff and number of items for each player are extracted from the output to compare the results.

Using a similar method as presented in the case of VRP, the VCG model was integrated into the simulator, and the results were evaluated for random layouts in terms of starting positions for players. The same number of iterations (1000) was used to compare the results in terms of optimal load balancing across a variable distribution of player locations on the map.

For visual inspection, the results were post-processed using a moving average filter as shown in Figure 13a for the average rewards and Figure 13b for the average number of assignments for each player type. We considered the same types of players with normalized reputations: 1 (truthful and accurate reporting), 0.8 (truthful and mostly accurate reporting) and 0.5 (false/inaccurate reporting).

The results show that both a higher number of tasks and a higher total reward are assigned for truthful bidding over the entire range of simulated layouts, which is directly influenced by the relative reputation of players.

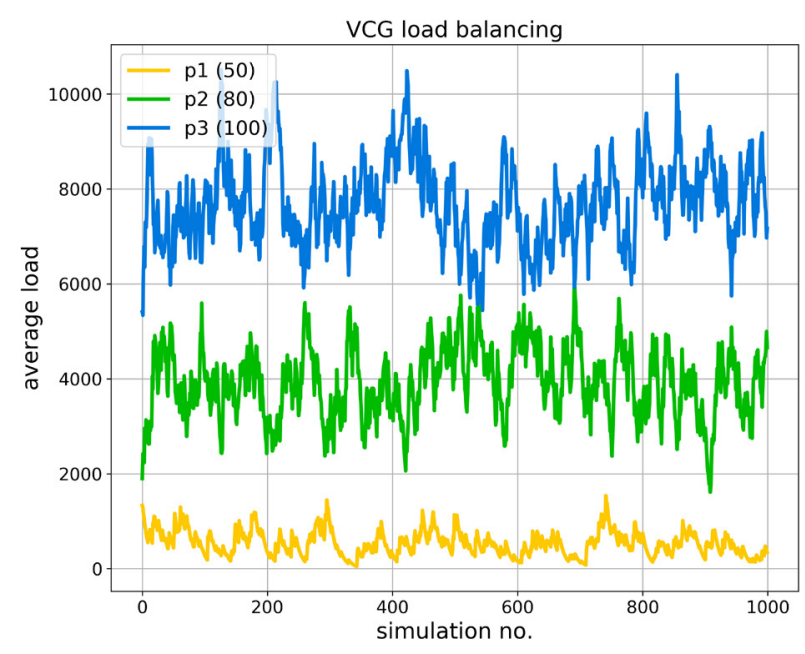

(a)

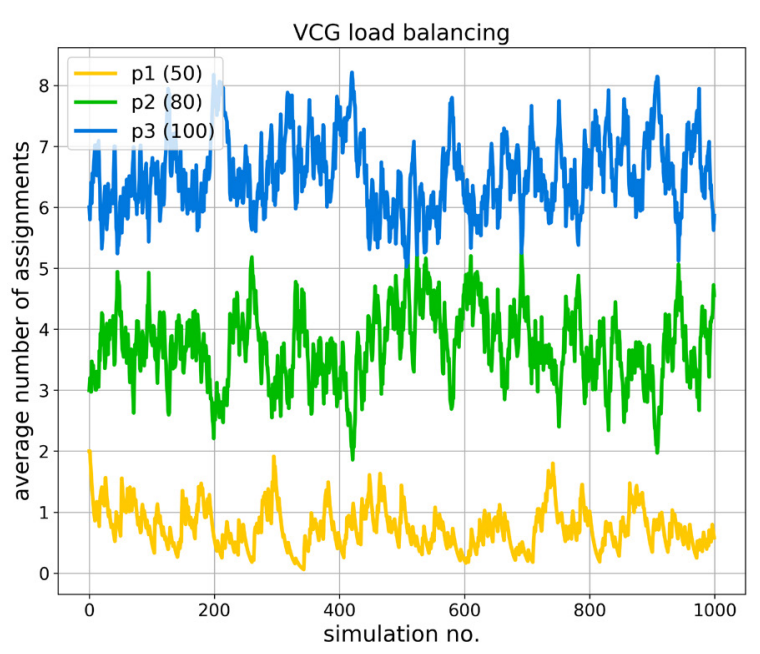

(b)

Figure 13. VCG load balancing; (a) average reward; (b) average number of assignments.

As the scenarios were generated based on a random distribution of players on the map, the results show a higher importance of player reputation with regards to the task allocation, while the proximity has a less pronounced influence in this sense.

Therefore, the reward scheme can include the reported cost (e.g., distance) to account for a decentralized, user-centric load balancing, in contrast to the VRP, where player locations must be processed by a centralized, i.e., platform-centric, routing service.

\subsection{Blockchain}

The blockchain framework is evaluated to see if the proposed data storage solution is feasible for the entire architecture. As mentioned before, Hyperledger is a new joiner in the blockchain technology stack and provides many plug and play functionalities, tools and studies about its characteristics.

According to [126], the performance of Fabric architecture is measured based on multiple metrics. All the tests were performed using a Hyperledger benchmark tool named Caliper [125] with the applied testing flow described in Figure 14. 


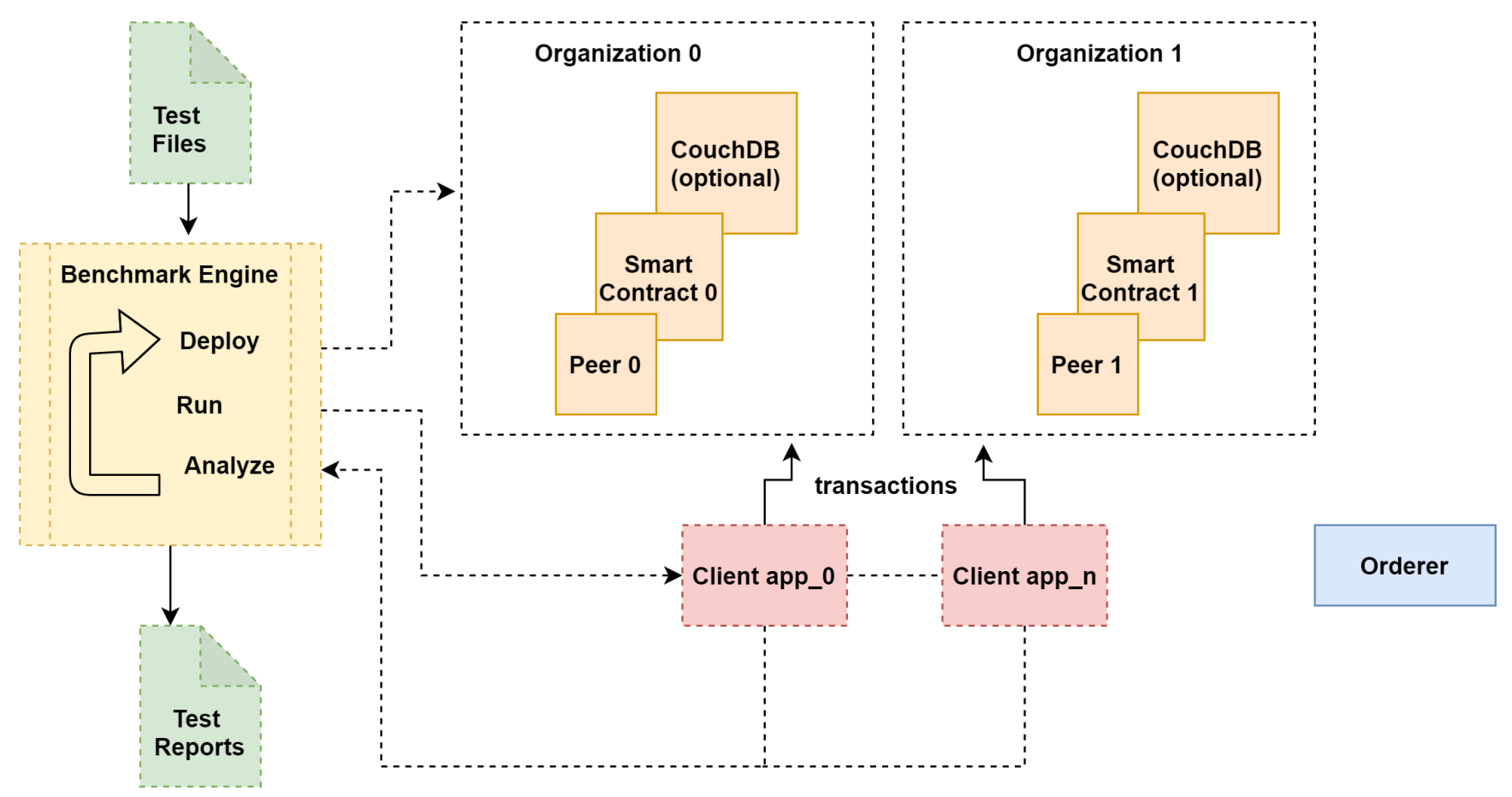

Figure 14. Blockchain test framework.

To better understand the results, the following key terms were defined: SUT (System Under Test), Read Latency (time between sending the read request and receiving the read response), Read Throughput (number of reading operations finished in a defined period), Transaction Latency (time from sending a new transaction to blockchain until the transaction is inserted and available to be retrieved) and Transaction Throughput (number of transactions added into blockchain in a defined period). The type of actions that were evaluated are the following:

- Write: creating a new transaction in the database (createAsset-single putState operation);

- Batch write: creating multiple batch transactions (createAssetsFromBatch-multiple putState operations);

- $\quad$ Read: get a transaction (getAsset—single getState operation);

- $\quad$ Batch read: get multiple transactions (getAssetsFromBatch-multiple getState operations).

The configuration parameters for Hyperledger Caliper [127] include the number of worker nodes, workload specification and dimensions (e.g., asset size, batch size), experiment duration and rate control mechanisms (e.g., fixed rate, fixed load), of which the most relevant were selected to provide a performance evaluation in terms of read/write operations on the blockchain.

Therefore, a fixed load rate controller was used with a specified constant load between 10 and 50 backlog transactions (i.e., pending confirmation), an asset size between 100B and $64 \mathrm{~KB}$ and a batch size between 1 and 50 to evaluate the proposed scenarios.

For the evaluation of read performance shown in Figure 15a, the fixed load was evaluated in terms of average latency, while increasing the asset size and using 10 worker nodes with a fixed load of 50 backlog transactions. The batch read performance shown in Figure $15 \mathrm{~b}$ was evaluated by increasing the batch size, using a predefined asset size of $8 \mathrm{~KB}$ and a fixed load of 20 backlog transactions. Similarly, the write performance shown in Figure 16a was evaluated using 5 worker nodes and a fixed load of 10 backlog transactions, and the batch write scenario shown in Figure $16 \mathrm{~b}$ was configured with a fixed $8 \mathrm{~KB}$ asset size and a fixed load of 5 backlog transactions.

According to the results in Figures 15 and 16, depending on the message size and the number of simultaneously read/write requests, the average time for retrieving/adding information from blockchain increases linearly with batch size and message size. 


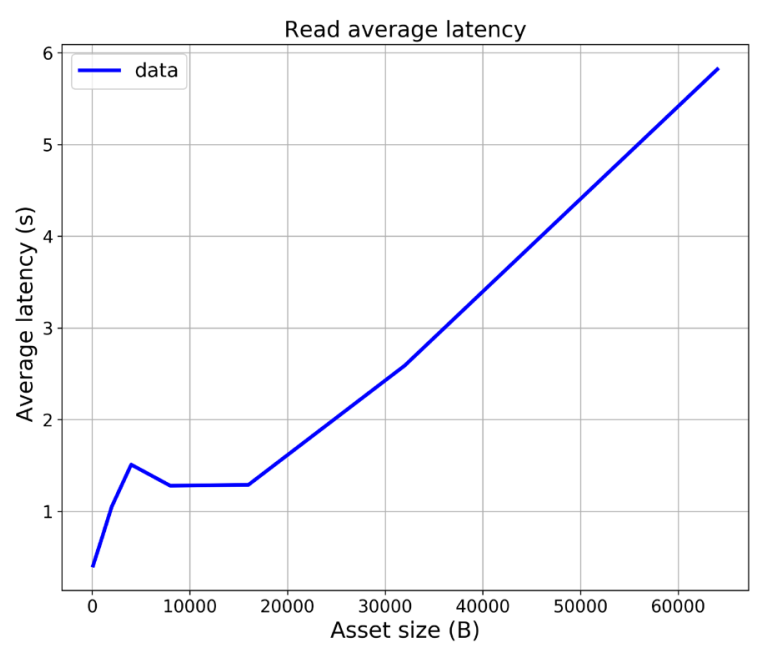

(a)

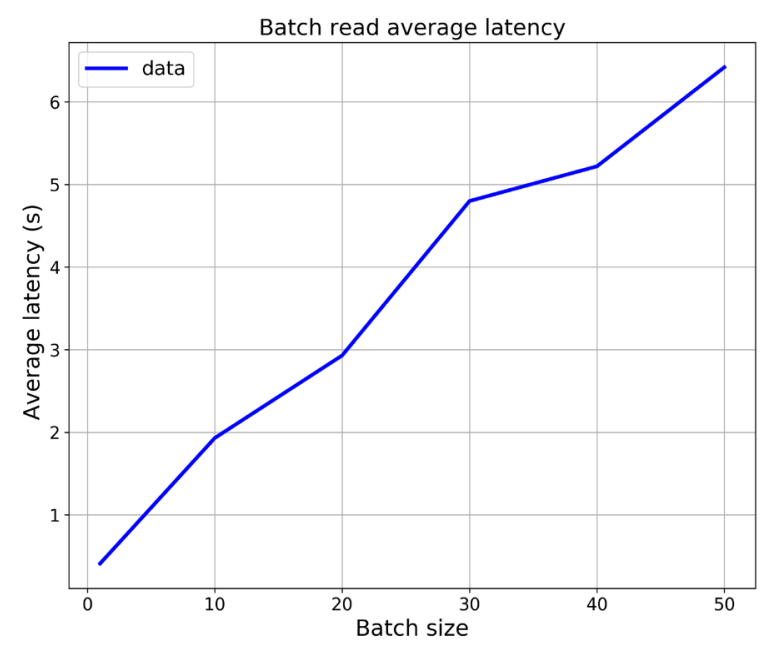

(b)

Figure 15. Read average latency (a) single read; (b) batch read.

The results show good performance and scalability with regards to the number of simultaneous requests and the message size when considering the test environment. Further small-scale experiments that were run multiple times have confirmed the accuracy of the results in terms of the overall conclusions, while the entire simulation is extremely resource-intensive and was only performed once.

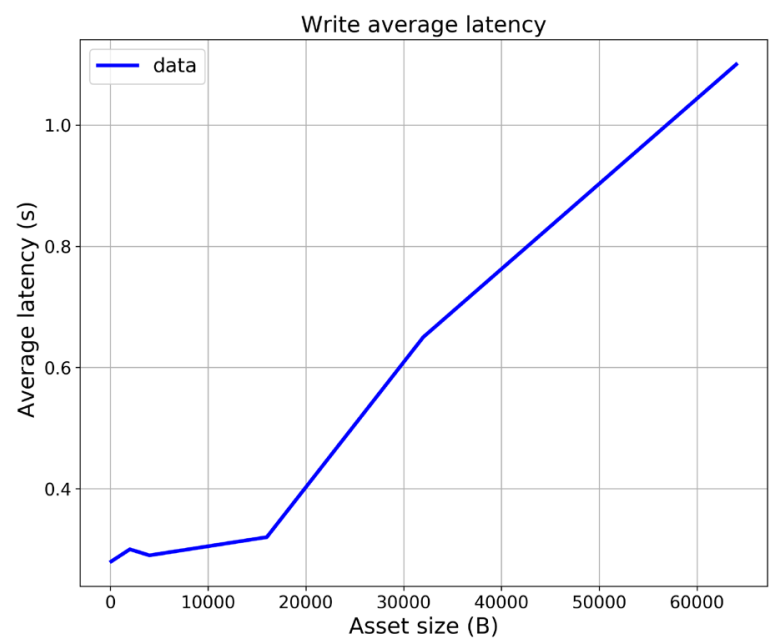

(a)

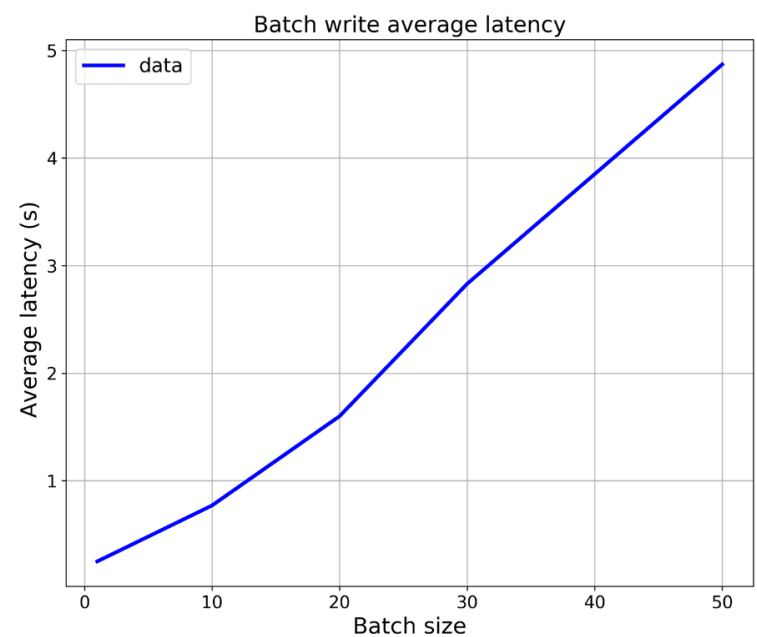

(b)

Figure 16. Write average latency (a) single write; (b) batch write.

\section{Discussion}

The simulation environment provides an evaluation of platform-centric and usercentric approaches to MCS. The task allocation and incentive mechanism represent two different problems that can be solved by either approach, while there are some specific aspects given by the geolocation component.

The player locations and distribution of tasks within a geographic area represent a key aspect in selecting the most suitable method for designing the incentive mechanism. In this sense, the VRP is a highly studied, industry-proven method that was adapted for this scenario and represents a platform-centric approach, while the VCG provides a user-centric reward mechanism based on player reputation in a reverse-auction model, where the actual 
sensing cost is reported by each player, instead of relying on a central dispatch system. The proposed solution offers great opportunities for further research by assessing both platform and user-oriented approaches.

Geolocation is a sensitive subject with regards to user privacy, which makes VCG a viable alternative for the reward-driven routing problem as well, demonstrated through the experimental results. While the location must be provided for georeferencing and map loading, the continuous tracking of user location not only can be resource-intensive, but may be prohibited by privacy laws. Therefore, an initial allocation of tasks is handled by the VRP model, while the dynamic reward mechanism is more accurately defined by the VCG model, where the actual sensing cost, i.e., distance traveled, is reported by each player.

A potential issue with crowdsensing is given by duplicate reporting, which can be addressed by a second reward factor based on the density of the reports within a geographic area in terms of physical location, category and timestamp. Moreover, by assigning lower rewards for clustered reports and higher rewards for outlier reports, the coverage of the proposed MCS solution can be improved.

Ambiguous reporting is another aspect that can be solved by integrating multiple validation schemes, i.e., using more accurate categories for reported issues and combined sensor data (e.g., camera, microphone) along with text-based reports. A more accurate rating system can further improve the level of interaction between the participants and encourage both truthful and accurate reporting.

While both platform-centric and user-centric models aim at increasing coverage, the integration in a real-time MCS platform based on the serious game implementation defined in this paper represents a challenge to be discussed in future research. The SG platform design is of primary importance when considering the actual involvement of citizens in solving water-related problems, with a game-driven mobile interface that is both practical and entertaining for the users. An additional aspect is increasing the environmental awareness of users, as well as an educational function.

The different stakeholders are assigned different roles, while the incentive mechanism is based on reputation and level of interaction with the real-world through gamified scenarios. The interactions are shaped by the incentive mechanisms and in-game economy for increased platform utility, i.e., optimal sensing capabilities.

The trust-based mechanism is supported by the Hyperledger Fabric architecture, which provides a scalable blockchain solution for audit-enhanced storage layer, with peer nodes represented by major stakeholders in water utility networks. The global implications for MCS, i.e., increasing the level of participation, can be found in multiple studies presented in Section 2, while an additional improvement in terms of overall platform utility is given by the reduced energy requirements for consensus when compared to other blockchains.

\section{Conclusions}

The gaming industry has come a long way since the inception of the Internet and game-related websites, based on community feedback and technical advancements. A brief history of PC and console games reveals a snapshot of a fast-growing multi-billion-dollar industry with multiple implications in culture and entertainment. Video games have basically changed the way we perceive and interact with the digital world, providing a bridge between humans and technology.

With the invention of smartphones and unified software distribution channels (i.e., app stores), mobile games represent a turning point for the gaming industry in the era of mobility. A data-driven survey has revealed a general trend for the traditional gaming industry (PC and console games), accounting for a decrease in average user scores for new releases, while mobile games have seen a steady increase in terms of user rating.

The MCS paradigm is based on the integration of mobile technologies into our lives and benefits from gamification to provide incentives for increasing the motivation and participation of citizens in urban water management. Existing solutions prove that there is 
a great deal of interest in MCS, while there are multiple challenges in designing an effective, citizen-centric platform for increasing the level of participation in the community. The motivation of users in MCS presents at least two different challenges that we addressed in this paper, represented by the practical value, i.e., optimal sensing capabilities, and entertainment value, i.e., serious gaming.

There are multiple challenges associated with MCS, with regards to the optimal sensing capabilities (e.g., optimal allocation of sensing tasks, truthful reporting, ensuring the quality of sensing data) and human motivation (e.g., attractive game design, the role of serious gaming, incentive mechanism design), and an effective design of MCS solutions requires a combination of game theory, mobile architecture and citizen science.

The SG-based MCS solution presented in this paper is based on the active participation of citizens in solving water-related problems. The literature review did not reveal a similar study that combines all the proposed components, whereas the effect of SG and Blockchain in MCS can be found in separate studies, some of which are related to the urban water context.

The methods discussed in this paper present two sides of the incentive mechanism design for successful MCS implementations. The mathematical models of distanceconstrained multi-depot vehicle routing problems with heterogeneous fleet capacity and Vickrey-Clarke-Groves auction are evaluated in the context of the proposed MCS scenario, with multiple initial conditions given by the location of players.

The mobile platform is designed as an interactive real-world game that aims to achieve a balance between platform utility and entertainment value based on the proposed crowdsensing model for solving water-related problems.

With blockchain support, the proposed solution covers some important aspects when designing secure and intelligent mobile systems, dealing with the inherent trust issues related to existing MCS solutions. The Hyperledger Fabric model was selected based on the privacy requirements in trust-based systems as well as the modularity achieved using smart contracts.

The background and integration of the proposed components in the context of CPSS is discussed in this paper, while further directions of research involve the deployment of an experimental platform and field testing with real users to evaluate the influence of different incentive strategies on the level of participation and quality of sensing data. An extensive study of the overall impact of each component on platform utility requires major investments for large-scale integration and marketing campaigns, while the proposed methodology provides a foundation for such developments.

Author Contributions: Conceptualization, A.P.; methodology, A.P., D.A. and B.P.; software, A.P. and B.P.; validation, A.P., D.A. and B.P.; formal analysis, A.P.; investigation, A.P. and B.P.; resources, A.P., D.A., B.P., M.M. and C.C.; data curation, A.P.; writing-original draft preparation, A.P., D.A. and B.P.; writing-review and editing, A.P., D.A., M.M. and C.C.; visualization, A.P. and D.A.; supervision, M.M. and C.C.; project administration, M.M. and C.C.; funding acquisition, M.M. and C.C. All authors have read and agreed to the published version of the manuscript.

Funding: This research was funded by UEFISCDI, grant number PN-III-P2-2.1-PED-2019-4993, Smart Urban Water-Based on Community Participation Through Gamification-Watergame Project.

Data Availability Statement: The data presented in this study are available in the GitHub repositories referenced in Section 6.

Acknowledgments: The mobile application described in this paper was developed with Leplace Global, a tech startup in the domain of augmented reality mobile games.

Conflicts of Interest: The authors declare no conflict of interest. 


\section{References}

1. Dressler, F. Cyber Physical Social Systems: Towards Deeply Integrated Hybridized Systems. In Proceedings of the 2018 International Conference on Computing, Networking and Communications (ICNC) IEEE, Maui, HI, USA, 5-8 March 2018; pp. $420-424$.

2. Rutten, M.; Minkman, E.; van der Sanden, M. How to Get and Keep Citizens Involved in Mobile Crowd Sensing for Water Management? A Review of Key Success Factors and Motivational Aspects. Wiley Interdiscip. Rev. Water 2017, 4, e1218. [CrossRef]

3. Silvertown, J. A New Dawn for Citizen Science. Trends Ecol. Evol. 2009, 24, 467-471. [CrossRef] [PubMed]

4. Nichols, R. The Video Game Business; International Screen Industries; British Film Institute: London, UK, 2014; ISBN 9781844573189.

5. Predescu, A.; Mocanu, M. Increasing Collaboration and Participation Through Serious Gaming for Improving the Quality of Service in Urban Water Infrastructure; Springer: Berlin, Germany, 2019; Volume 373 LNBIP, ISBN 9783030366902.

6. Friedlmaier, M.; Tumasjan, A.; Welpe, I.M. Disrupting Industries with Blockchain: The Industry, Venture Capital Funding, and Regional Distribution of Blockchain Ventures, Proceedings of the 51st Annual Hawaii International Conference on System Sciences (HICSS), Waikoloa, HI, USA, 3-6 January 2018; SSRN: Amsterdam, The Netherlands, 2018.

7. Swan, M. Blockchain: Blueprint for a New Economy; O’Reilly Media Inc.: Newton, MA, USA, 2015; ISBN 978-1491920497.

8. Keitsch, M. Structuring Ethical Interpretations of the Sustainable Development Goals—Concepts, Implications and Progress. Sustainability 2018, 10, 829. [CrossRef]

9. Manion, M. Ethics, Engineering, and Sustainable Development. IEEE Technol. Soc. Mag. 2002, 21, 39-48. [CrossRef]

10. Guo, B.; Yu, Z.; Zhou, X.; Zhang, D. From Participatory Sensing to Mobile Crowd Sensing. In Proceedings of the 2014 IEEE International Conference on Pervasive Computing and Communication Workshops (PERCOM WORKSHOPS) IEEE, Budapest, Hungary, 24-28 March 2014; pp. 593-598.

11. Ganti, R.K.; Ye, F.; Lei, H. Mobile Crowdsensing: Current State and Future Challenges. IEEE Commun. Mag. 2011, 49, 32-39. [CrossRef]

12. Pouryazdanpanah, M.; Kantarci, B.; Dasari, V. On the Impact of Selective Data Acquisition in Mobile Crowd-Sensing Performance. In Proceedings of the 2018 IEEE Canadian Conference on Electrical \& Computer Engineering (CCECE) IEEE, Quebec City, QC, Canada, 13-16 May 2018.

13. Wang, Y.; Yan, Z.; Feng, W.; Liu, S. Privacy Protection in Mobile Crowd Sensing: A Survey. World Wide Web 2020, $23,421-452$. [CrossRef]

14. Dasari, V.; Kantarci, B.; Pouryazdan, M.; Foschini, L.; Girolami, M. Game Theory in Mobile CrowdSensing: A Comprehensive Survey. Sensors 2020, 20, 2055. [CrossRef] [PubMed]

15. Jiang, L.-Y.; He, F.; Wang, Y.; Sun, L.-J.; Huang, H. Quality-Aware Incentive Mechanism for Mobile Crowd Sensing. J. Sensors 2017, 2017, 5757125. [CrossRef]

16. Attia, P. The Full History of Board Games. Available online: https://medium.com/@peterattia/the-full-history-of-board-games -5e622811ce89 (accessed on 12 December 2020).

17. Wallach, O. 50 Years of Gaming History, by Revenue Stream (1970-2020). Available online: https://www.visualcapitalist.com/50years-gaming-history-revenue-stream/ (accessed on 10 December 2020).

18. Dillon, R. The Golden Age of Video Games: The Birth of a Multibillion Dollar Industry; CRC Press: Boca Raton, FL, USA, 2011; ISBN 9781439873236.

19. Shliakhovchuk, E.; Muñoz García, A. Intercultural Perspective on Impact of Video Games on Players: Insights from a Systematic Review of Recent Literature. Educ. Sci. Theory Pract. 2020, 20, 40-58. [CrossRef]

20. Garcia-Fernandez, J.; Medeiros, L. Cultural Heritage and Communication through Simulation Videogames-A Validation of Minecraft. Heritage 2019, 2, 2262-2274. [CrossRef]

21. Wilcox-Netepczuk, D. Immersion and Realism in Video Games-The Confused Moniker of Video Game Engrossment. In Proceedings of the 2013 18th International Conference on Computer Games: AI, Animation, Mobile, Interactive Multimedia, Educational \& Serious Games (CGAMES) IEEE, Los Alamitos, CA, USA, 30 July-1 August 2013; pp. $92-95$.

22. Garda, B.M. Nostalgia in Retro Game Design. In Proceedings of the DiGRA '13-Proceedings of the 2013 DiGRA International Conference: DeFragging Game Studies, Atlanta, GA, USA, 26-29 August 2014.

23. Mäyrä, F. Mobile Games. Int. Encycl. Digit. Commun. Soc. 2015, 1-6. [CrossRef]

24. Jin, D.Y. Mobile Gaming in Asia: Politics, Culture and Emerging Technologies; Springer: Cham, Switzerland, 2016; ISBN 978-94-024-0824-9.

25. Mansell, R.; Ang, P.H.; Steinfield, C.; van der Graaf, S.; Ballon, P.; Kerr, A.; Ivory, J.D.; Braman, S.; Kleine, D.; Grimshaw, D.J. The International Encyclopedia of Digital Communication and Society, 3 Volume Set; ICAZ-Wiley Blackwell-ICA International Encyclopedias of Communication; Wiley: Hoboken, NJ, USA, 2015; ISBN 9781118290743.

26. Carvalho, M.; Bellotti, F.; Berta, R.; De Gloria, A.; Islas Sedano, C.; Hauge, J.; Hu, J.; Rauterberg, M. An Activity Theory-Based Model for Serious Games Analysis and Conceptual Design. Comput. Educ. 2015, 87, 166-181. [CrossRef]

27. Loh, C.; Yanyan, S.; Ifenthaler, D. Serious Games Analytics: Theoretical Framework. In Serious Games Analytics. Advances in Game-Based Learning; Springer: Cham, Switzerland, 2015; pp. 3-30, ISBN 978-3-319-05833-7.

28. Weiner, M.G. An Introduction to War Games; RAND Corporation: Santa Monica, CA, USA, 1959.

29. Loucks, D.P.; van Beek, E. Water Resource Systems Planning and Management; Springer International Publishing: Cham, Switzerland, 2017; ISBN 978-3-319-44232-7.

30. Mayer, I. The Gaming of Policy and the Politics of Gaming: A Review. Simul. Gaming 2009, 40, 825-862. [CrossRef] 
31. Clark, C.A. Serious Games; University Press of America: Lanham, MD, USA, 2002; ISBN 978-0819161482.

32. Duke, R. Origin and Evolution of Policy Simulation: A Personal Journey; SAGE: Newcastle upon Tyne, UK, 2011 ; Volume 42.

33. Medema, W.; Furber, A.; Adamowski, J.; Zhou, Q.; Mayer, I. Exploring the Potential Impact of Serious Games on Social Learning and Stakeholder Collaborations for Transboundary Watershed Management of the St. Lawrence River Basin. Water 2016, 8, 175. [CrossRef]

34. Rijcken, T.; Stijnen, J.; Slootjes, N. "SimDelta"-Inquiry into an Internet-Based Interactive Model for Water Infrastructure Development in The Netherlands. Water 2012, 4, 295-320. [CrossRef]

35. Gleick, P.H. Environment and Security: Water Conflict Chronology-Version 2000. In The World's Water 2000-2001; Island Press: Washington, DC, USA, 2000.

36. Palmer, R.N.; Cardwell, H.E.; Lorie, M.A.; Werick, W. Disciplined Planning, Structured Participation, and Collaborative ModelingApplying Shared Vision Planning to Water Resources. JAWRA J. Am. Water Resour. Assoc. 2013, 49, 614-628. [CrossRef]

37. Vörösmarty, C.J.; Green, P.; Salisbury, J.; Lammers, R.B. Global Water Resources: Vulnerability from Climate Change and Population Growth. Science 2000, 289, 284-288. [CrossRef]

38. Lin, Y.-P.; Petway, J.; Anthony, J.; Mukhtar, H.; Liao, S.-W.; Chou, C.-F.; Ho, Y.-F. Blockchain: The Evolutionary Next Step for ICT E-Agriculture. Environments 2017, 4, 50. [CrossRef]

39. Hutson, M. Can Bitcoin's Cryptographic Technology Help Save the Environment? Available online: https://www.sciencemag.o $\mathrm{rg} /$ news /2017/05/can-bitcoin-s-cryptographic-technology-help-save-environment (accessed on 20 December 2020).

40. Bashir, I. Mastering Blockchain; Packt: Birmingham, UK, 2017; ISBN 9781787125445.

41. Acharya, V.; Yerrapati, A.E.; Prakash, N. Oracle Blockchain Quick Start Guide; Packt: Birmingham, UK, $2019 ;$ ISBN 9781789804164.

42. Antonopoulos, A.M. The Internet of Money, 1st ed.; Merkle Bloom LLC: Columbia, MD, USA, 2016; ISBN 1537000454.

43. EMA NEWS \& INSIGHTS Blockchain and the Water Industry-Smart Contracts. Available online: https://www.ema-inc.com/ news-insights/2018/10/blockchain-and-the-water-industry-smart-contracts/ (accessed on 20 December 2020).

44. Hargrave, S.J.; Karnoupakis, E. Blockchain Success Stories; O’Reilly Media Inc.: Newton, MA, USA, 2020; ISBN 9781098114824.

45. Wood, G. Ethereum: A Secure Decentralised Generalised Transaction Ledger; Elsevier: Amsterdam, The Netherlands, 2014.

46. "Smart Contracts" Are the Future of Blockchain. Available online: https://www.americanbanker.com/opinion/smart-contracts -are-the-future-of-blockchain (accessed on 20 December 2020).

47. Sajana, P.; Sindhu, M.; Sethumadhavan, M. On Blockchain Applications: Hyperledger Fabric And Ethereum. Int. J. Pure Appl. Math. 2018, 118, 2965-2970.

48. Gates, M. Blockchain: Ultimate Guide to Understanding Blockchain, Bitcoin, Cryptocurrencies, Smart Contracts and the Future of Money; CreateSpace Independent Publishing Platform: Scotts Valley, CA, USA, 2017; ISBN 978-1547090686.

49. Zamora, W.; Calafate, C.T.; Cano, J.-C.; Manzoni, P. A Survey on Smartphone-Based Crowdsensing Solutions. Mob. Inf. Syst. 2016, 2016, 9681842. [CrossRef]

50. Mrazovic, P. Crowdsensing-Driven Route Optimisation Algorithms for Smart Urban Mobility; KTH Royal Institute of Technology: Stockholm, Sweden, 2018; ISBN 9789177299479.

51. Wan, J.; Liu, J.; Shao, Z.; Vasilakos, A.V.; Imran, M.; Zhou, K. Mobile Crowd Sensing for Traffic Prediction in Internet of Vehicles. Sensors 2016, 16, 88. [CrossRef]

52. Luo, T.T.; Kanhere, S.S.; Huang, J.; Das, S.K.; Wu, F. Sustainable Incentives for Mobile Crowdsensing: Auctions, Lotteries, and Trust and Reputation Systems. IEEE Commun. Magaz. 2017, 55, 68-74. [CrossRef]

53. Liu, X.; Ota, K.; Liu, A.; Chen, Z. An Incentive Game Based Evolutionary Model for Crowd Sensing Networks. Peer-to-Peer Netw. Appl. 2016, 9, 692-711. [CrossRef]

54. Morris, S.; Shadmehr, M. Reward and Punishment in a Regime Change Game. SSRN Electron. J. 2017. [CrossRef]

55. Li, T.; Jung, T.; Li, H.; Cao, L.; Wang, W.; Li, X.; Wang, Y. Scalable Privacy-Preserving Participant Selection in Mobile Crowd Sensing. In Proceedings of the 2017 IEEE International Conference on Pervasive Computing and Communications (PerCom), Kona, HI, USA, 13-17 March 2017; pp. 59-68.

56. Tao, X.; Song, W. Efficient Path Planning and Truthful Incentive Mechanism Design for Mobile Crowdsensing. Sensors 2018, 18, 4408. [CrossRef]

57. Sessa, P.G.; Walton, N.; Kamgarpour, M. Exploring the Vickrey-Clarke-Groves Mechanism for Electricity Markets. IFACPapersOnLine 2017, 50, 189-194. [CrossRef]

58. Nekouei, E.; Alpcan, T.; Chattopadhyay, D. Game-Theoretic Frameworks for Demand Response in Electricity Markets. IEEE Trans. Smart Grid 2015, 6, 748-758. [CrossRef]

59. Lam, A.Y.S. Combinatorial Auction-Based Pricing for Multi-Tenant Autonomous Vehicle Public Transportation System. IEEE Trans. Intell. Transp. Syst. 2016, 17, 859-869. [CrossRef]

60. Roca-Riu, M.; Menendez, M. The Potential of Flexible Reservations in a Car Sharing System with an Auction Scheme. IEEE Access 2019, 7, 154627-154639. [CrossRef]

61. Hershberger, J.; Suri, S. Vickrey Pricing in Network Routing: Fast Payment Computation. In Proceedings of the 42nd IEEE Symposium on Foundations of Computer Science, Washington, DC, USA, 8-10 October 2001; pp. 252-259.

62. Zhou, H.; Leung, K.; Li, V.O.K. Auction-Based Schemes for Multipath Routing in Selfish Networks. In Proceedings of the 2013 IEEE Wireless Communications and Networking Conference (WCNC) IEEE, Shanghai, China, 7-10 April 2013; pp. $1956-1961$. 
63. Xu, Z.; Mei, L.; Choo, K.-K.R.; Lv, Z.; Hu, C.; Luo, X.; Liu, Y. Mobile Crowd Sensing of Human-like Intelligence Using Social Sensors: A Survey. Neurocomputing 2018, 279, 3-10. [CrossRef]

64. Xiong, J.; Ma, R.; Chen, L.; Tian, Y.; Lin, L.; Jin, B. Achieving Incentive, Security, and Scalable Privacy Protection in Mobile Crowdsensing Services. Wirel. Commun. Mob. Comput. 2018, 2018, 8959635. [CrossRef]

65. Zhang, J.; Wang, D. Duplicate Report Detection in Urban Crowdsensing Applications for Smart City. In Proceedings of the 2015 IEEE International Conference on Smart City/SocialCom/SustainCom (SmartCity) IEEE, Chengdu, China, 19-21 December 2015; pp. 101-107.

66. Prisner, E. Game Theory through Examples; MAA Press: Washington, DC, USA, 2014; ISBN 978-1-61444-115-1.

67. Mayer, I. Towards a Comprehensive Methodology for the Research and Evaluation of Serious Games. Proc. Comput. Sci. 2012, 15, 233-247. [CrossRef]

68. Talasila, M.; Curtmola, R.; Borcea, C. Alien vs. Mobile User Game: Fast and Efficient Area Coverage in Crowdsensing, Proceedings of the 2014 6th International Conference on Mobile Computing, Applications and Services (MobiCASE), Austin, TX, USA, 6-7 November 2014; IEEE Computer Society: Los Alamitos, CA, USA, November 2014; pp. 65-74.

69. Serious Games. Available online: https:/ / www.genevawaterhub.org/resource/serious-games (accessed on 28 November 2020).

70. Tygron EN Geodesign Platform. Available online: http://www.tygron.com/ (accessed on 28 November 2020).

71. Gaberdan, E.; Maier, K.; Söbke, H.; Londong, J. Illustrating Decisions to End-Users: The Case of Agent-Based Simulation for Stormwater Management. In Proceedings of the Computational Methods in Water Resources 2014 XX International Conference, Stuttgart, Germany, 10-13 June 2014.

72. Sureephong, P.; Puritat, K.; Chernbumroong, S. Enhancing User Performance and Engagement through Gamification: Case Study of Aqua Republica. In Proceedings of the 2016 10th International Conference on Software, Knowledge, Information Management Applications (SKIMA) IEEE, Chengdu, China, 15-17 December 2016; pp. 220-224.

73. Aqua Republica. Available online: http:/ /aquarepublica.com/ (accessed on 28 November 2020).

74. Danish Hydraulic Institute. Mike Hydro Basin: A Multipurpose, Map Based Decision Support Tool for Integrated River Basin Analysis, Planning and Management-User Guide; Danish Hydraulic Institute: Hørsholm, Denmark, 2014.

75. Chew, C.; Zabel, A.; Lloyd, G.J.; Gunawardana, I.; Monninkhoff, B. A Serious Gaming Approach for Serious Stakeholder Participation. In Proceedings of the International Conference on Hydroinformatics, New York, NY, USA, 17-21 August 2014.

76. Douven, W.; Mul, M.L.; Son, L.; Bakker, N.; Radosevich, G.; Hendriks, A. Games to Create Awareness and Design Policies for Transboundary Cooperation in River Basins: Lessons from the Shariva Game of the Mekong River Commission. Water Resour. Manag. 2014, 28, 1431-1447. [CrossRef]

77. Valkering, P.; van der Brugge, R.; Offermans, A.; Haasnoot, M.; Vreugdenhil, H. A Perspective-Based Simulation Game to Explore Future Pathways of a Water-Society System under Climate Change. Simul. Gaming 2013, 44, 366-390. [CrossRef]

78. Seibert, J.; Vis, M.J.P. Irrigania-A Web-Based Game about Sharing Water Resources. Hydrol. Earth Syst. Sci. 2012, 16, 2523-2530. [CrossRef]

79. Bassi, A.; Rego, F.; Harrisson, J.; Lombardi, N. WATERSTORY ILE: A Systemic Approach to Solve a Long-Lasting and Far-Reaching Problem. Simul. Gaming 2015, 46, 404-429. [CrossRef]

80. Van Alphen, J. The Delta Programme and Updated Flood Risk Management Policies in the Netherlands. J. Flood Risk Manag. 2016, 9, 310-319. [CrossRef]

81. Rijcken, T.; Christopher, D.K. SimDelta Global: Towards a Standardised Interactive Model for Water Infrastructure Development. Eur. J. Geogr. 2013, 4, 6-21.

82. Hill, H.; Hadarits, M.; Rieger, R.; Strickert, G.; Davies, E.G.R.; Strobbe, K.M. The Invitational Drought Tournament: What Is It and Why Is It a Useful Tool for Drought Preparedness and Adaptation? Weather Clim. Extrem. 2014, 3, 107-116. [CrossRef]

83. Wu, Y.; Song, P.; Wang, F. Hybrid Consensus Algorithm Optimization: A Mathematical Method Based on POS and PBFT and Its Application in Blockchain. Math. Probl. Eng. 2020, 2020, 13. [CrossRef]

84. Sankar, L.S.; Sindhu, M.; Sethumadhavan, M. Survey of Consensus Protocols on Blockchain Applications. In Proceedings of the 2017 4th International Conference on Advanced Computing and Communication Systems (ICACCS), Coimbatore, India, 6-7 January 2017; pp. 1-5.

85. Extance, A. Could Bitcoin Technology Help Science? Available online: https://www.nature.com/articles/d41586-017-08589-4 (accessed on 15 December 2020).

86. Jaramillo, F.; Destouni, G. Local Flow Regulation and Irrigation Raise Global Human Water Consumption and Footprint. Available online: https:/ / science.sciencemag.org/content/350/6265/1248 (accessed on 15 December 2020).

87. Bordel, B.; Martin, D.; Alcarria, R.; Robles, T. A Blockchain-Based Water Control System for the Automatic Management of Irrigation Communities. In Proceedings of the 2019 IEEE International Conference on Consumer Electronics (ICCE) IEEE, Las Vegas, NV, USA, 11-13 January 2019; pp. 1-2.

88. Garg, P. Can Blockchain Technology Help Us Solve the Water Management Crisis? Available online: https://btcmanager.com/c an-blockchain-technology-help-us-solve-the-water-management-crisis / (accessed on 15 December 2020).

89. iotWorm How the Internet of Things Helps in Water Management. Available online: https://iotworm.com/internet-things-help s-water-management-system/ (accessed on 15 December 2020).

90. Dogo, E.; Salami, A.; Nwulu, N.; Aigbavboa, C. Blockchain and Internet of Things-Based Technologies for Intelligent Water Management System. Artif. Intell. IoT. Trans. Comput. Sci. Comput. Intell. 2019, 129-150. [CrossRef] 
91. Sarp, S.; Lee, S.; Jeon, D.J.; Kim, J.H. Smart Water Grid: The Future Water Management Platform. Desalin. Water Treat. 2014, 55, 339-346. [CrossRef]

92. Mutchek, M.; Williams, E. Moving Towards Sustainable and Resilient Smart Water Grids. Challenges 2014, 5, 123-137. [CrossRef]

93. Reyna, A.; Martín, C.; Chen, J.; Soler, E.; Díaz, M. On Blockchain and Its Integration with IoT. Challenges and Opportunities. Futur. Gener. Comput. Syst. 2018, 88, 173-190. [CrossRef]

94. Robles, T.; Alcarria, R.; Martín, D.; Navarro, M.; Calero, R.; Iglesias, S.; López, M. An IoT Based Reference Architecture for Smart Water Management Processes. Wirel. Mob. Networks, Ubiquitous Comput. Dependable Appl. 2015, 6, 4-23.

95. Mendonca, S.; Junior, J.S.; Alencar, F. The Blockchain-Based Internet of Things Development: Initiatives and Challenges. In Proceedings of the ICSEA 2017, The Twelfth International Conference on Software Engineering Advances, Athens, Greece, 8-12 October 2017; pp. 28-33.

96. Poberezhna, A. Chapter 14-Addressing Water Sustainability with Blockchain Technology and Green Finance. In Transforming Climate Finance and Green Investment with Blockchains; Marke, A., Ed.; Academic Press: Cambridge, MA, USA, 2018; pp. 189-196, ISBN 978-0-12-814447-3.

97. Pincheira, M.; Vecchio, M.; Giaffreda, R.; Kanhere, S.S. Cost-Effective IoT Devices as Trustworthy Data Sources for a BlockchainBased Water Management System in Precision Agriculture. Comput. Electron. Agric. 2021, 180, 105889. [CrossRef]

98. Grigoras, G.; Bizon, N.; Enescu, F.M.; Guede, J.M.L.; Salado, G.F.; Brennan, R.; O’Driscoll, C.; Dinka, M.O.; Alalm, M.G. ICT Based Smart Management Solution to Realize Water and Energy Savings through Energy Efficiency Measures in Water Distribution Systems. In Proceedings of the 2018 10th International Conference on Electronics, Computers and Artificial Intelligence (ECAI) IEEE, Iasi, Romania, 28-30 June 2018; pp. 1-4.

99. Rottondi, C.; Verticale, G. A Privacy-Friendly Gaming Framework in Smart Electricity and Water Grids. IEEE Access 2017, 5, 14221-14233. [CrossRef]

100. Khutsoane, O.; Isong, B.; Abu-Mahfouz, A.M. IoT Devices and Applications Based on LoRa/LoRaWAN. In Proceedings of the IECON 2017-43rd Annual Conference of the IEEE Industrial Electronics Society, Beijing, China, 29 October-1 November 2017; pp. 6107-6112.

101. Wang, S.; Taha, A.F.; Wang, J. Blockchain-Assisted Crowdsourced Energy Systems. In Proceedings of the 2018 IEEE Power \& Energy Society General Meeting (PESGM) IEEE, Portland, OR, USA, 5-9 August 2018; pp. 1-5.

102. Yang, M.; Zhu, T.; Liang, K.; Zhou, W.; Deng, R.H. A Blockchain-Based Location Privacy-Preserving Crowdsensing System. Futur. Gener. Comput. Syst. 2019, 94, 408-418. [CrossRef]

103. Kadadha, M.; Otrok, H.; Mizouni, R.; Singh, S.; Ouali, A. SenseChain: A Blockchain-Based Crowdsensing Framework for Multiple Requesters and Multiple Workers. Futur. Gener. Comput. Syst. 2020, 105, 650-664. [CrossRef]

104. Jia, B.; Zhou, T.; Li, W.; Liu, Z.; Zhang, J. A Blockchain-Based Location Privacy Protection Incentive Mechanism in Crowd Sensing Networks. Sensors 2018, 18, 3894. [CrossRef] [PubMed]

105. Predescu, A.; Mocanu, M. A Data Driven Survey of Video Games. In Proceedings of the 202012 th International Conference on Electronics, Computers and Artificial Intelligence (ECAI) IEEE, Bucharest, Romania, 25-27 June 2020; pp. 1-6.

106. Poh, M. Evolution of Home Video Game Consoles: 1967-2011. Available online: https://www.hongkiat.com/blog/evolution-of -home-video-game-consoles-1967-2011/ (accessed on 18 December 2020).

107. Blue Flame Labs MobyGames: Game Browser. Available online: https://www.mobygames.com/browse/games (accessed on 12 December 2020).

108. Tlili, T.; Faiz, S.; Krichen, S. A Hybrid Metaheuristic for the Distance-Constrained Capacitated Vehicle Routing Problem. Procedia Soc. Behav. Sci. 2014, 109, 779-783. [CrossRef]

109. Hanum, F.; Hartono, A.P.; Bakhtiar, T. On the Multiple Depots Vehicle Routing Problem with Heterogeneous Fleet Capacity and Velocity. IOP Conf. Ser. Mater. Sci. Eng. 2018, 332, 12052. [CrossRef]

110. Toro, E.; Escobar Zuluaga, A.; Granada-Echeverri, M. Literature Review on the Vehicle Routing Problem in the Green Transportation Context. Luna Azul 2015, 362-387. [CrossRef]

111. Zhang, W.; Gajpal, Y.; Appadoo, S.; Wei, Q. Others Multi-Depot Green Vehicle Routing Problem to Minimize Carbon Emissions. Sustainability 2020, 12, 3500. [CrossRef]

112. Makowski, L.; Ostroy, J.M. Vickrey-Clarke-Groves Mechanisms and Perfect Competition. J. Econ. Theory 1987, 42, $244-261$. [CrossRef]

113. Stanford University 3.3 VCG: Examples-Efficient Mechanisms. Available online: https://www.coursera.org/lecture/game-theo ry-2/3-3-vcg-examples-42beq (accessed on 14 December 2020).

114. Blummer, T.; Sean, M.B.; Cachin, C. An Introduction to Hyperledger; Hyperledger Organization: San Francisco, CA, USA, 2018.

115. Hyperledger Architecture Working Group and others Hyperledger Architecture Volume 1: Introduction to Hyperledger Business Blockchain Design Philosophy and Consensus; Hyperledger Organization: San Francisco, CA, USA, 2017.

116. Ongaro, D.; Ousterhout, J. In Search of an Understandable Consensus Algorithm. In Proceedings of the 2014 USENIX Annual Technical Conference, USENIX Association. Philadelphia, PA, USA, 19-20 June 2014; pp. 305-319.

117. Hyperledger The Ordering Service. Available online: https://hyperledger-fabric.readthedocs.io/en/release-2.2/orderer/order ing_service.html (accessed on 20 December 2020). 
118. Arsene, D.; Pahonţu, B.; Predescu, A.; Mocanu, M.; Lupu, C. A Hyperledger Integration for Audit-Enhanced Decision Support in a Smart Water Distribution System. In Proceedings of the 2020 IEEE 16th International Conference on Intelligent Computer Communication and Processing (ICCP), IEEE, Cluj-Napoca, Romania, 3-5 September 2020; pp. 499-504.

119. LEPLACE GLOBAL Urban Experience / Leplace. Available online: https:/ / www.leplace.online/ (accessed on 18 December 2020).

120. Google Vehicle Routing Problem I OR-Tools I Google Developers. Available online: https://developers.google.com/optimization /routing/vrp (accessed on 14 December 2020).

121. Google Overview I Directions API I Google Developers. Available online: https://developers.google.com/maps/documentation / directions / overview (accessed on 14 December 2020).

122. Shan, K. Python Code for Implementing a Vickrey-Clarke-Groves Auction [GitHub Repository]. 2018. Available online: https://github.com/kqshan/vcg-auction (accessed on 26 January 2021).

123. Predescu, A. Crowdsensing Model Simulator [GitHub Repository]. 2020. Available online: https://github.com/alexp25/crowd sensing (accessed on 26 January 2021).

124. Predescu, A. Blockchain Benchmark for Hyperledger Fabric [GitHub Repository]. 2021. Available online: https://github.com/a lexp25/blockchain-bench (accessed on 26 January 2021).

125. Lincoln, N. Hyperledger Caliper. Available online: https:// hyperledger.github.io/caliper/ (accessed on 20 December 2020).

126. The Hyperledger White Paper Working Group Hyperledger Blockchain Performance Metrics. Available online: https://www.hy perledger.org/learn/publications/blockchain-performance-metrics (accessed on 20 December 2020).

127. Hyperledger Caliper Benchmark Configuration. Available online: https://hyperledger.github.io/caliper/v0.4.2/bench-config/ (accessed on 26 January 2021). 